\title{
Phantom Validation of Polyp Automatic Detection using Microwave Endoscopy for Colorectal Cancer Prevention and Early Detection
}

\author{
Alejandra Garrido \\ MiWEndo Solutions S.L. \\ Roberto Sont \\ MiWEndo Solutions S.L. \\ Walid Dghoughi \\ MiWEndo Solutions S.L. \\ Sergi Marcoval \\ MiWEndo Solutions S.L. \\ Jordi Romeu \\ Universitat Politècnica de Catalunya \\ Glòria Fernández-Esparrach \\ Hospital Clínic, University of Barcelona, CIBEREHD. IDIBAPS. Barcelona \\ Ignasi Belda \\ MiWEndo Solutions S.L. \\ Marta Guardiola ( $\nabla$ marta@miwendo.com ) \\ MiWEndo Solutions S.L.
}

\section{Research Article}

Keywords: colonoscopy, optical colonoscopy, sensitivity and specificity, colorectal cancer (CRC)

Posted Date: May 6th, 2021

DOI: https://doi.org/10.21203/rs.3.rs-479847/v1

License: (1) (1) This work is licensed under a Creative Commons Attribution 4.0 International License.

Read Full License

Version of Record: A version of this preprint was published at IEEE Access on January 1st, 2021. See the published version at https://doi.org/10.1109/ACCESS.2021.3124019. 


\title{
Phantom Validation of Polyp Automatic Detection using Microwave Endoscopy for Colorectal Cancer Prevention and Early Detection
}

\author{
Alejandra Garrido1,+, Roberto Sont $1,+$, Walid Dghoughi $1,+$, Sergi Marcoval1,+, \\ Jordi Romeu2, Glòria Fernández-Esparrach1,3, Ignasi Belda1, and Marta \\ Guardiola1, ${ }^{*}$
}

1MiWEndo Solutions, Barcelona, 08014, Spain

2Universitat Politècnica de Catalunya, CommSensLab, Barcelona, 08034, Spain

3Endoscopy Unit, Gastroenterology Department, Hospital Clínic, University of Barcelona. CIBEREHD.

IDIBAPS. Barcelona, 08036, Spain

*marta@miwendo.com

these authors contributed equally to this work

\begin{abstract}
A system to integrate microwave imaging with optical colonoscopy is presented. The overarching goal is to improve the prevention, diagnosis and understanding of one of the main health and economic burdens of an increasingly ageing population that is colorectal cancer. Our system can address the two main challenges of colonoscopy improve the detection of precancerous lesions called polyps and classify them according to their malignancy - and moves forward a major trend of the field that is automating medical explorations. To do so, a complete imaging system able to emit an alarm when a polyp is detected is designed, manufactured and validated with a realistic colon phantom composed by tissue-mimicking oil-gelatin materials reproducing the anatomy and dielectric properties of a human colon with a polyp. The acquisition is done by a miniaturized ring-shaped switched array of 16 antennas attachable at the tip of a conventional colonoscope. It has been conceived to satisfy endoscopy size restrictions, patient safety and intercompatibility with the current clinical practice. A Modified Monofocusing imaging method preceded by a previous frame average subtraction as calibration technique shows perfect detection of a 10-mm polyp (100\% sensitivity and specificity) in the two analyzed trajectories. Results of realistic phantom demonstrate the feasibility of the system in future preclinical trials.
\end{abstract}

\section{Introduction}

Globally, 1.8 million new cases of colorectal cancer (CRC) are diagnosed annually, and 861,000 people died from CRC in 2018 ${ }^{1}$. This leads to CRC being the second most common cause of cancer death. CRC describes cancerous malignancies of the colon and rectum. Most cases of CRC begin as a growth of tissue, known as a polyp, which originates in the lining of the colon or rectum and grows in size over a period of several years. Polyps are common in patients over 50 years, but certain polyps (known as adenomas) are estimated to be precursors to the $90 \%$ of CRC cases $^{2}$. The overall five-year survival rate of CRC is approximately $65 \%$, but drops to a $14.3 \%$ if diagnosed at advanced stages that is quite common given the absence of remarkable symptoms when the cancer is in a localized phase ${ }^{3}$.

Large-scale studies have found that CRC can be cured in more than $90 \%$ of cases if adenomas are removed $^{2}$. Optical colonoscopy is the most effective method for CRC diagnosis and the only method able to remove polyps in the entire colon. During a colonoscopy, a long, flexible tube called colonoscope is inserted into the rectum. A tiny video camera at the tip of the tube allows the doctor to view the inside of the entire colon. However, the limited field of view of the camera -less than $180^{\circ}-$, inadequate colon preparation and the subjectivity of the exploration dependent on the endoscopist's experience, among others, result in a polyp miss rate of $22 \%{ }^{4}$ and a risk of developing cancer after a negative colonoscopy -the so-called interval CRC- of $8 \% 5$. Moreover, there is no accurate objective method to differentiate benign and malignant polyps, which leads to an inefficient treatment of patients in economic and time management terms. Nowadays $23 \%$ of colonoscopies are repeated and all the polyps are resected and histologically analyzed, even if they are benign, which overloads endoscopy and pathology departments. 
The two major challenges in CRC early diagnosis are to improve polyp detection and to classify polyps according to their pathology. Technologically advanced endoscopes and add-on accessories are being used to widen colon visualization and improve polyp or adenoma detection rate with different levels of performance $^{6}$, such as high resolution colonoscopes, colonoscopes with multiple cameras, and caps to spread mucosa. Chromoendoscopy and endoscopic microscopy using endocytoscopy and endomicroscopy are methods developed to magnify, enhance and estimate mucosa tissue and cell characteristics that can be linked to malignancy. All these techniques are all restricted to the optical information captured by the camera of the endoscope, none of these technical innovations can determine the malignity of the tissues based on a quantitative indicator and all depend on the operator ability and experience. Related to the trend of automatizing the detection, artificial intelligence is increasingly used for real-time assessment of endoscopic images ${ }^{7}$. However, being able to offer a reliable and practical solution for endoscopists that does not overload the visualization screen still persists. Furthermore, the effectiveness of these methods is again limited by the field of view of the optical cameras, as recent studies have shown that deficient coverage is one of the reasons for missed polyps ${ }^{8}$.

Microwave imaging has shown promise as a portable, non-ionizing, low power, non-invasive and potentially low-cost medical diagnostic method $^{9}$. Microwave imaging is able to obtain anatomical and functional images of the interior of the human body representing the existing contrast in dielectric properties - the relative permittivity and the conductivity- between different tissues. Dielectric properties are biomarkers of many health problems such as ostheoporosis ${ }^{10}$, heart infarction ${ }^{11}$, pulmonary edema, etc., being breast cancer ${ }^{12,13}$ and brain stroke ${ }^{14,15}$ the most researched and advanced topics. We recently demonstrated that the dielectric properties correlate with the malignancy grade of colon polyps ${ }^{16}$-the more similar to cancer, the higher the relative permittivity and conductivity-. Results indicate that the maximum contrast between healthy mucosa and cancer was $30 \%$ and $90 \%$ for the relative permittivity and conductivity, respectively, at $8 \mathrm{GHz}$.

This paper reports a complete system to integrate microwave imaging with optical colonoscopy aimed to improve the prevention and early detection of colorectal cancer. To the best of our knowledge, this is the first time that microwave imaging is proposed for endoscopic applications. With the proposed system, the detection of polyps can be automatized by emitting an alarm when a polyp is detected to warn the endoscopist. This feature will fill the gap between endoscopists with different levels of experience, which has been demonstrated to play an important role in colonoscopy effectiveness. Microwave imaging is able to form $360^{\circ}$ images with a fair trade-off between resolution and tissue penetration. Therefore, it has the potential to both reduce the visualization problems of conventional colonoscopy due to poor colon coverage and provide differential diagnosis during colorectal examinations. In this way, doctors will have quantitative information to make more informed decisions, for example about whether or not to remove a polyp.

The proposed acquisition system has to adapt and be robust to established colonoscopy clinical practice, which entails a series of peculiarities with respect to previous microwave imaging systems reported in the literature $^{17,18}$. As an advantage, there is no need to penetrate the body since polyps are superficial lesions. However, a number of challenges have emerged. On the one hand, space restrictions limit the number of antennas in the array, reduces the size and thickness of each antenna element and increases mutual coupling between them. Electrically small antennas are narrowband, limiting the range of imaging algorithms to frequency domain ones. On the other hand, the antennas are distributed in a circular array enclosed by the imaging region instead of the imaging region being surrounded by the antennas, that is the usual configuration for microwave imaging. Finally, the unknown and changing distance from the imaging region with respect to the antenna array increases the degrees of freedom introduced by the uncertainty of the spatial and temporal delay reception of the scattered fields. So, calibration methods to eliminate all unwanted effects other than the target -the polyp- are essential. The system is verified with a colon phantom that simulates a realistic colonoscopy exploration. The phantom models a section of colon with polyps. The phantom is composed of gelatin-oil based materials to mimic the dielectric properties of colon healthy mucosa and polyps. The reconstructed images from the phantom are quantitatively analyzed and the efficacy of the calibration and detection threshold are investigated.

\section{Results}

\section{Microwave endoscopy system}

The imaging system consists of: 1) a cylindrical ring-shaped acquisition device that can be attached to the tip of a conventional colonoscope; and 2) an external unit with a microwave transceiver, a controlling and 
a processing unit. The acquisition device is connected via cables to the external unit. The acquisition device contains two switched arrays of eight antennas organized in two rings, one containing the transmitters and the other the receivers. The external unit is composed by: 1) a vector network analyzer (VNA) employed to generate and measure the microwave signals which are transmitted through the switching system to the selected transmitting and receiving antenna pair; 2) a laptop to process the measured data; and 3) a microcontroller used to generate the switch's control signals. Error! Reference source not found. shows the block diagram of the proposed system. The acquisition device and the external unit are connected via two slim 1.13-mm coaxial cables that transmit the radiofrequency signal, and eight copper cables to control de switches. Each switch is controlled by a three bits' code of 0-5 V signals.

The antenna elements are cavity backed slot antennas as described in our previous paper ${ }^{19}$. So that the array can take a cylindrical shape, the antennas have been assembled on a polyamide flexible printed circuit board that contains the microstrip feeding lines and the two radiofrequency switches. The flexible assembly is wrapped around a cylindrical 3D printed piece that contains the cavities to house the antennas on the outside and has a hole inside to introduce the colonoscope. Miniature connectors are used for a non-bulky final assembly. The whole set is covered with a biocompatible heat shrinkable material to protect the circuit from moisture and the patient from lesions. The final dimensions of the acquisition device are $30 \mathrm{~mm}$ in length by $20 \mathrm{~mm}$ in diameter, having a total thickness of $3 \mathrm{~mm}$. The dimensions and shape of the device ensure non-obstruction of the front tip of the colonoscope, avoids camera concealment, injuring the patient or hindering the maneuverability of the colonoscope.
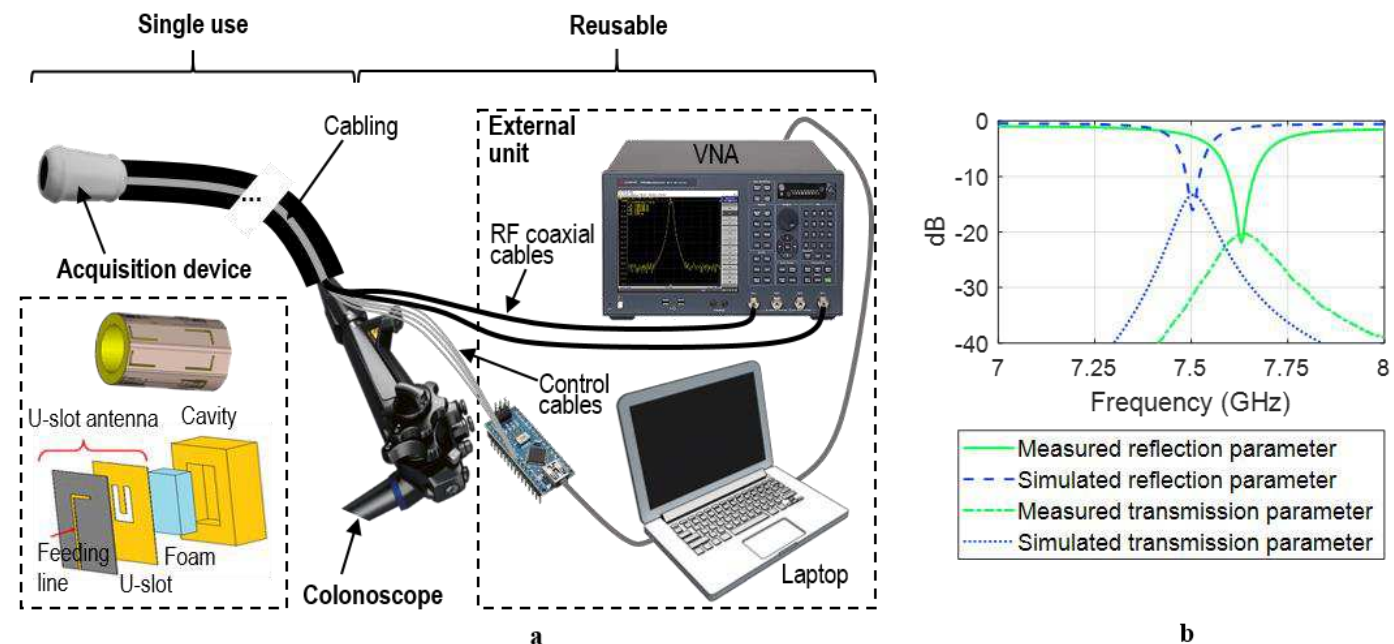

Figure 1. (a) Imaging system consists of a cylindrical ring-shaped acquisition device attached to the tip of a colonoscope connected via cables to the external unit. The external unit consists of a vector network analyzer, a microcontroller and a laptop. (b) Comparison between the measured and simulated reflection and transmission coefficients.

It is noted from Error! Reference source not found.(b) that the antenna operates over the band 7.6-7.66 $\mathrm{GHz}$, which is approximately $0.8 \%$ fractional bandwidth with respect to the center frequency of 7.63 $\mathrm{GHz}$, assuming the $-10 \mathrm{~dB}$ reflection coefficient as the reference. The reason for such a small bandwidth is the miniaturization of the antenna with dimensions $0.12 \lambda \times 0.16 \lambda$. Since the antennas are very close together, mutual coupling increases, introducing error in the measured scattered field. Cavity-backed antennas have been used to minimize coupling to $-13 \mathrm{~dB}$. The comparison between the measured and the simulated results shows a slight frequency shift and increased losses in measured results. The main difference between simulated and measured results is the higher losses in the measurement, that are clearly seen on both reflection and transmission coefficients. These losses are produced mainly by the metallization roughness and the welding, which is not considered in the simulation. To obtain the measurement results at the antenna plane and remove the effects of the long cables, a Thru, Reflect, Line (TRL) calibration is used.

\section{Colon phantom measurement testbed}

\section{Colon with polyps' phantom}

The colon is the last part of the gastrointestinal tract. It has a segmented appearance due to a series of folds called haustra and it its about $150 \mathrm{~cm}$ long and 9-4 $\mathrm{mm}$ in diameter. Polyps are slow-growing overgrowths of the colonic mucosa protruding into the lumen. When a colonoscopy is performed, the 
colon lumen is expanded using carbon dioxide insufflation. To model a colon during colonoscopy, we have built a phantom with the anatomical and electrical properties of an expanded human colon. Our phantom is composed of a colon lumen model made of expanded polystyrene of $268 \mathrm{~mm}$ in length and $66.7 \mathrm{~mm}$ in diameter, as shown in Figure 2. The colon lumen is placed in the center of a cylindrical methacrylate box of $300 \mathrm{~mm}$ in length and $150 \mathrm{~mm}$ in diameter. The expanded polystyrene has the same dielectric properties as air, making it an excellent model for the colon lumen. The polystyrene surface is undulated to simulate colon haustra and contains 4 holes to place polyps at different positions. The space in between the colon lumen and the box walls is filled with a gelatin-oil mixture mimicking the dielectric properties of healthy mucosa tissue. In this paper, a spherical polyp of $10 \mathrm{~mm}$ in diameter was placed in the position Polyp 3 shown in Figure 2. The polyp is composed of a gelatin-oil mixture mimicking the dielectric properties of a polyp with high grade dysplasia (HGD) tissue ${ }^{16}$.

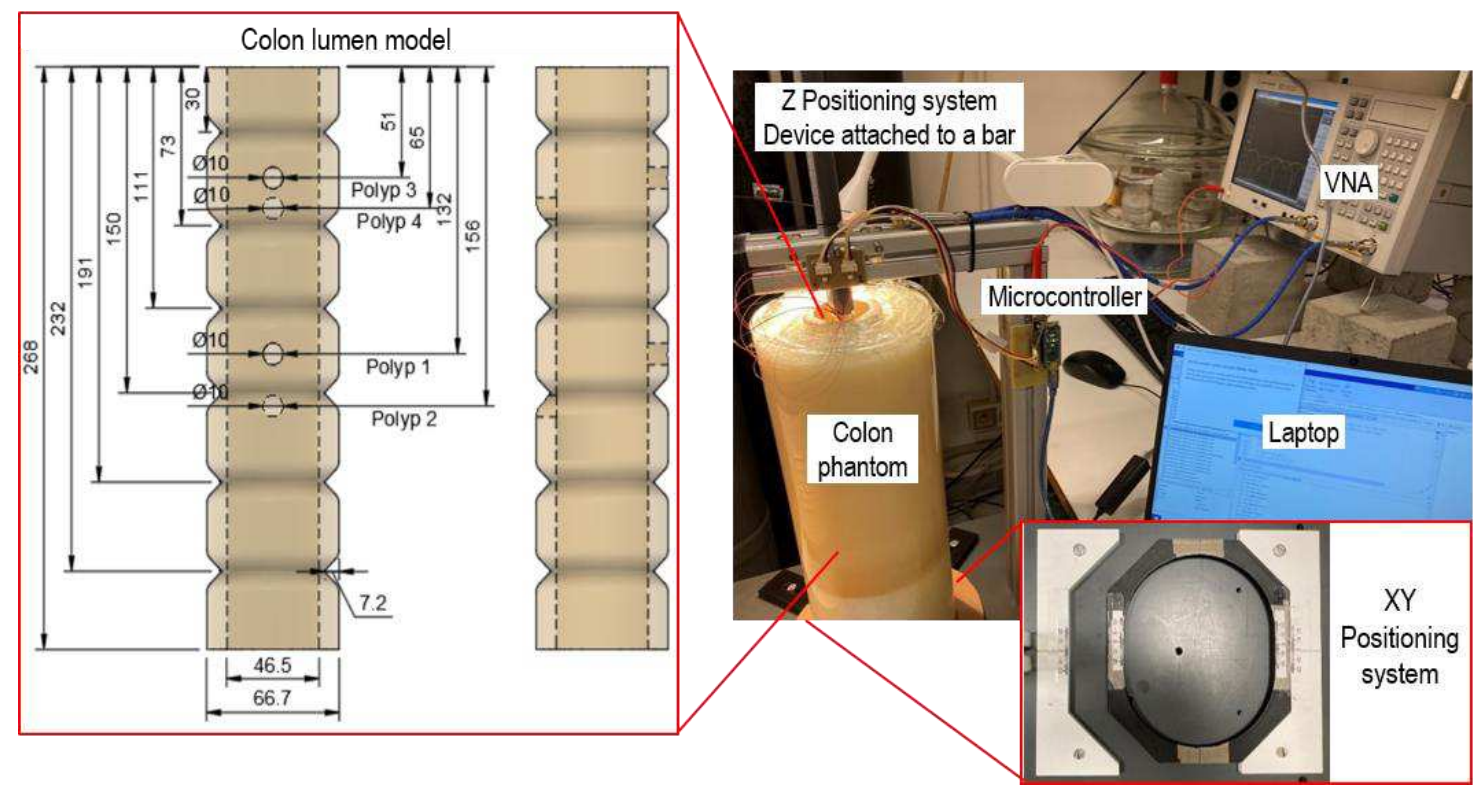

Figure 2. Measurement setup. The acquisition device is attached to the tip of a plastic bar. The colon phantom is placed on a $3 D$ positioning system. The colon phantom is composed by tissue-mimicking materials poured in a methacrylate cylindrical box and an expanded polystyrene colon lumen model. The external microwave transceiver, controlling and processing unit is composed by a vector network analyser (VNA), a microcontroller, a laptop.

The dielectric properties of the tissue-mimicking materials were measured using the keysight N1501A Dielectric slim form Probe Kit with the N1500A Materials Measurement Software Suite connected to a vector network analyzer Keysight E5071C. The dielectric properties of the materials were monitored throughout the measurement campaign. Figure 3(a) shows the time evolution of the dielectric properties of the tissue-mimicking materials. The plot includes the mean and standard deviation of three consecutive measurements at $7.6 \mathrm{GHz}$. The materials remained stable over 40 days by preserving them into the fridge covered with kitchen film to prevent it from drying out or losing consistency. Figure 3(b) presents the relative permittivity and conductivity of the tissue-mimicking materials corresponding to the healthy mucosa and the polyp with high grade dysplasia compared to the previously measured dielectric properties of the corresponding human tissues ${ }^{16}$. It can be observed that the dielectric properties of the tissue-mimicking materials reproduce both the relative permittivity and conductivity of the human tissue dielectric properties at $7.6 \mathrm{GHz}$. 


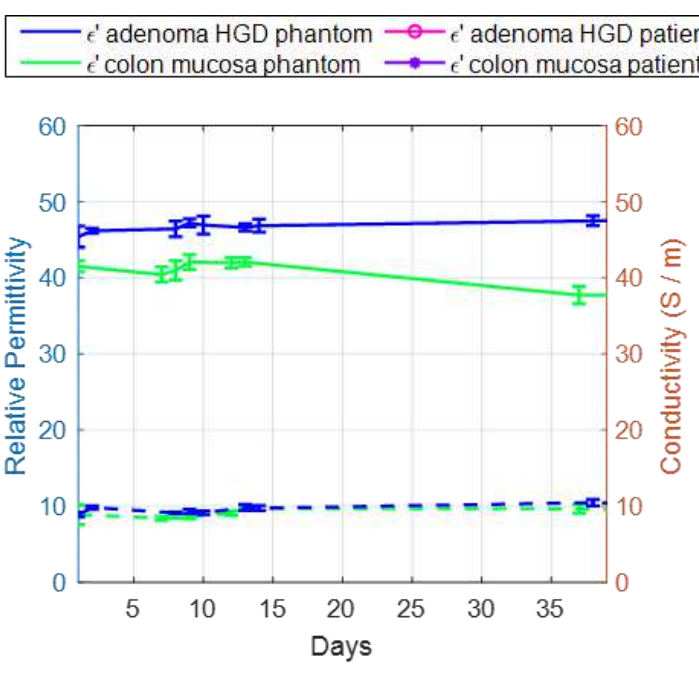

a

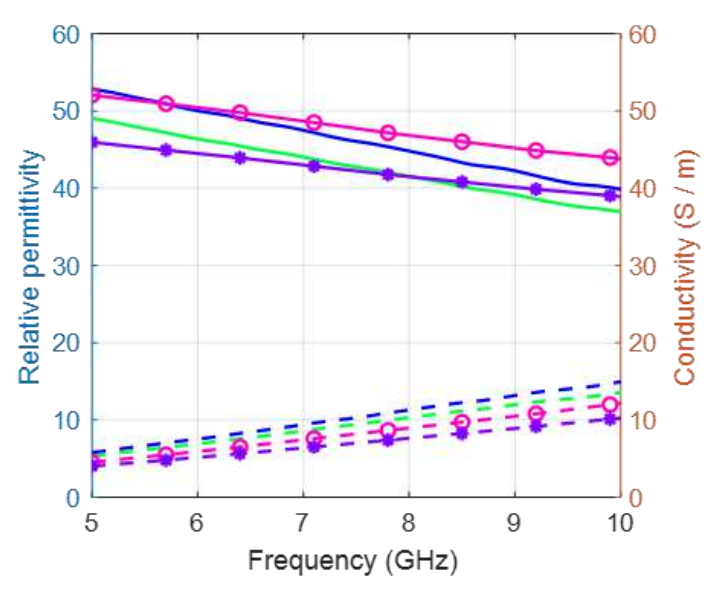

b

Figure 3. Dielectric properties of the tissue-mimicking materials used for the phantom. (a) time evolution of the average and standard deviation of the measured relative permittivity $\left(\epsilon^{\prime}\right)$ and conductivity $(\sigma)$ of the adenoma with high grade dysplasia's (HGD) phantom and the colon mucosa's phantom materials at 7.6 GHz, (b) measured relative permittivity and conductivity of the phantom compared to the real patient tissues over the frequency.

\section{Measurement setup}

To be able to reproduce a real colonoscopy exploration while the position of the acquisition device is known, a 3D positioning system has been built, see Figure 2. The measurement setup is composed by an L-shaped metallic structure fixed on a plastic base. The metallic structure holds a plastic bar equipped with a ruler. The acquisition device is attached at the tip of the plastic bar. With this bar, the device is introduced vertically inside the colon lumen model, and is moved along it to take the measurements of the transmission coefficient over the frequency band of interest along a predefined trajectory. The colon phantom is located on a mobile platform that allows it to move it along $\mathrm{x}$ and $\mathrm{y}$ directions, and the plastic bar allows it to move the acquisition device vertically along $\mathrm{z}$ direction. In this way a complete positioning system in three dimensions is achieved, enabling to model realistic trajectories inside the colon phantom.

\section{Experimental detection results}

Following data acquisition and processing, the results of the colon phantom are obtained using the different calibration and focusing techniques proposed in the methods section. Figure 4 shows the evolution of the normalized maximum amplitude of the reconstructed image registered in each step of the trajectory. The trajectories analyzed in Figure 4 correspond to the Trajectory 1 and Trajectory 2, where the polyp is between the frames [44,47] and [41,44], respectively. The raw measured transmission coefficients for each antenna combination have been calibrated using three different strategies: The Total Average Temporal Subtraction, the Hop and N Average Temporal Subtraction and the Healthy Average Temporal Subtraction. From now on, they will be referred to as AverageTotal, Hop\&NAverage and AverageHealthy respectively. The first two strategies intend to emulate the results of the AverageHealthy that is an ideal and nonrealistic calibration method that relies on averaging the signals measured in the healthy colon portions and, subsequently, subtracting them from each measurement frame. For each calibration strategy, the image has been created using both Bifocusing and Modified Monofocusing focusing algorithms. The movement along the y-axis of the device, or the distance to the polyp, has also been included in the plots in order to analyze the relation between the device's displacement and the reconstruction. Vertical continuous orange lines indicate the limits of colon haustrum and vertical continuous red lines indicate the limits of the polyp.

The maximum reconstructed amplitude represented in Figure 4 has been normalized between 0 and 1, using the maximum amplitude of each calibration set, to enable a better comparison between the different methods and trajectories. It is possible to appreciate that the evolution of the maximum registered along the trajectory reveals an identical tendency for the three different calibration strategies and for the two focusing algorithms, Modified Monofocusing and Bifocusing. Indeed, the absolute maximum of all the traces displayed in Figure 4 corresponds to the position of the polyp. Local maximums are also observed 
when the trajectory approaches the colon walls. Haustrum do not seem to produce any effect on the reconstructed results.
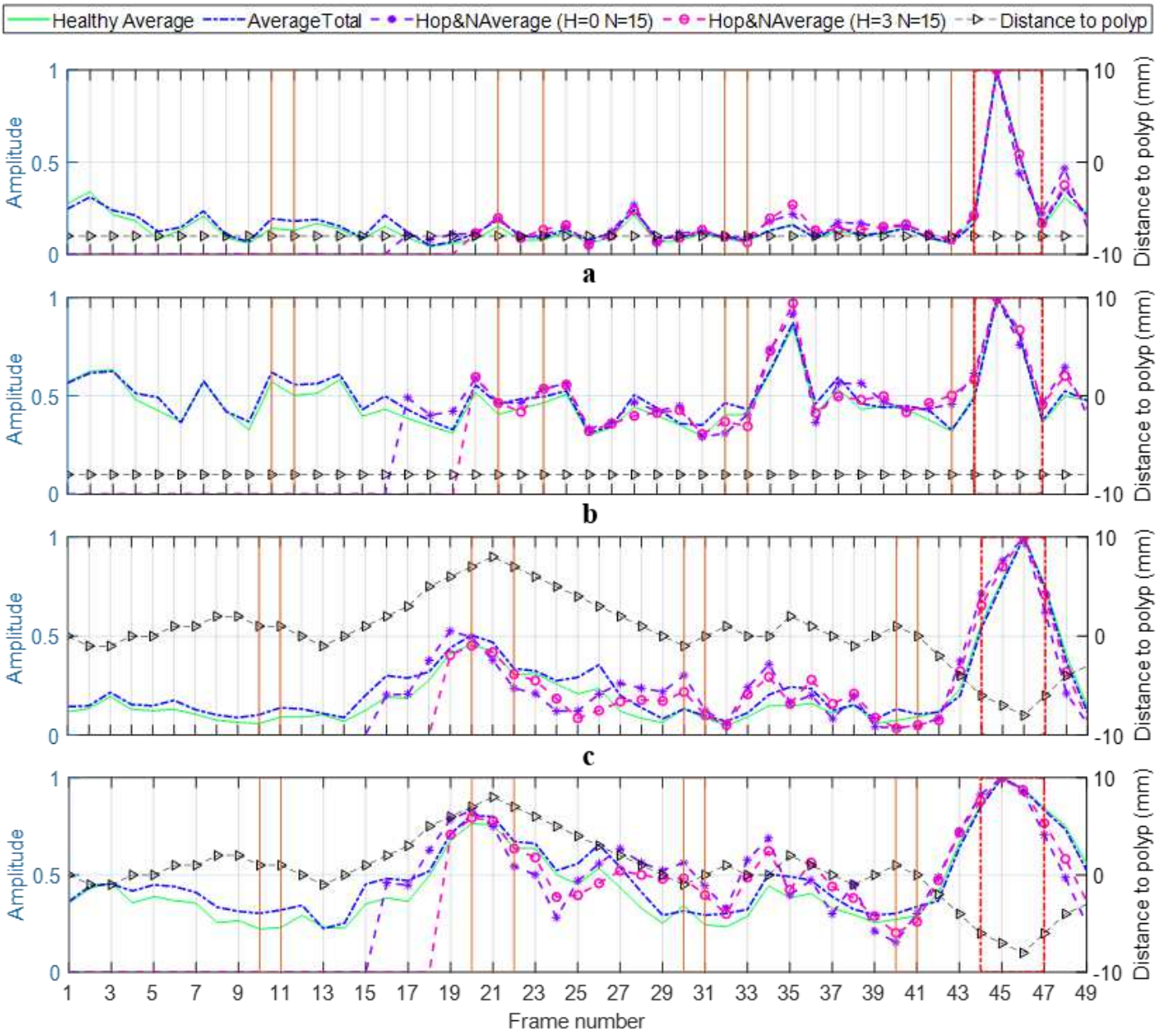

d

Figure 4. Evolution of the normalized maximum amplitude registered in each frame of the trajectories after applying the different calibration and focusing strategies proposed. a) Trajectory 1 using Modified Monofocusing, $b$ ) Trajectory 1 using Bifocusing, c) Trajectory 2 using Modified Monofocusing, and d) Trajectory 2 using Bifocusing algorithm.

Concerning calibration strategies, both AverageTotal and Hop\&NAverage, offer a similar performance to the Healthy Average Temporal Subtraction. In the case of the Hop\&NAverage strategy, the number of averaged frames $(N)$ is set to 15 and two different sizes of the backwards leap $(H)$ have been analyzed. On the first hand, $H$ has been assigned a value of 3 since the polyp's diameter that has been used in both trajectories is equal to $10 \mathrm{~mm}$ and, therefore, there are only 4 frames in which the polyp is inside the detection zone of the device. Hence, if we want to avoid including information from de polyp in the calibration set, it is necessary to omit the 3 previous frames to the frame that is actually reconstructed. Likewise, the case of $H=0$ has also been analyzed to assess if a sufficiently large number of averaged frames $(N)$ could minimize the effect of the polyps during the calibration, emulating, hence, the principle of the AverageTotal strategy. Figure 5Figure 4 includes the results obtained when calibrating with different $N$ values and $\mathrm{H}=0$. As shown in Figure 5 the larger $N$ is, the less sensitive the calibration is to noise and therefore the more robust it is. Additionally, in the frames where the polyp is detected, a larger $N$ minimizes the contribution of the polyp in the average used to calibrate, allowing a more similar detection to the one observed with the AverageTotal strategy. 


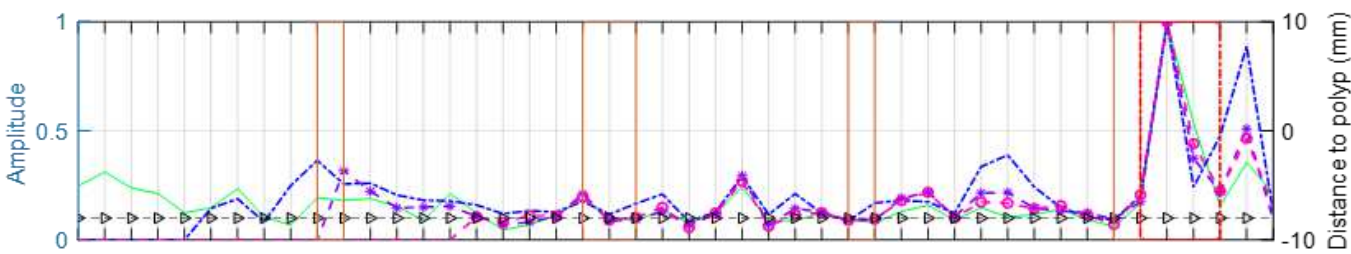

a

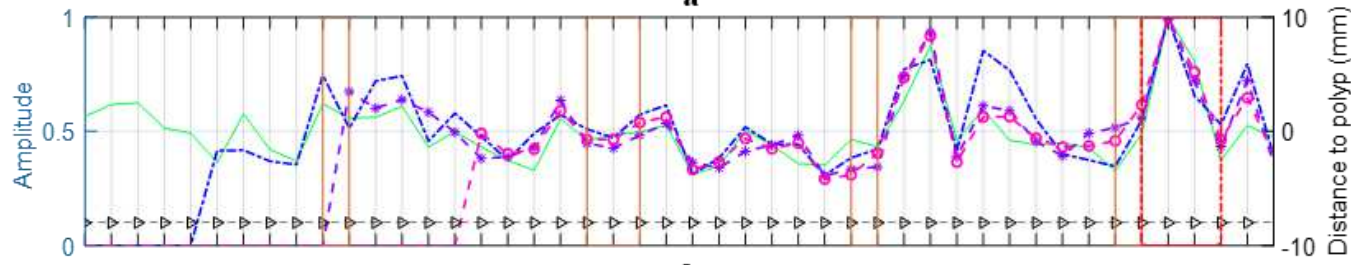

b

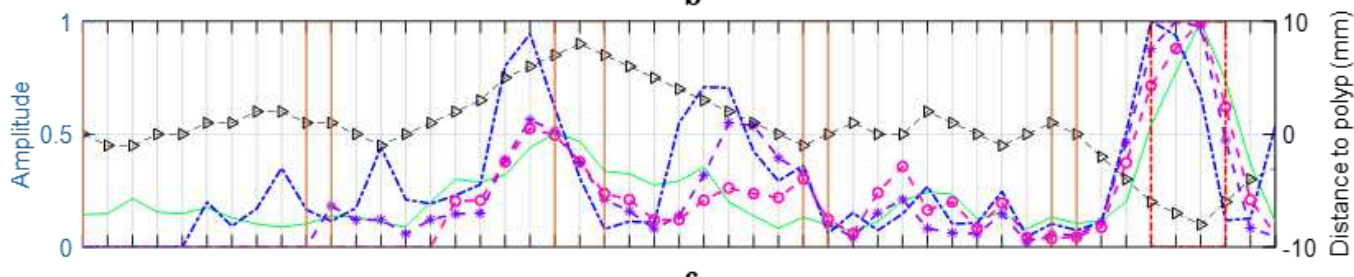

C

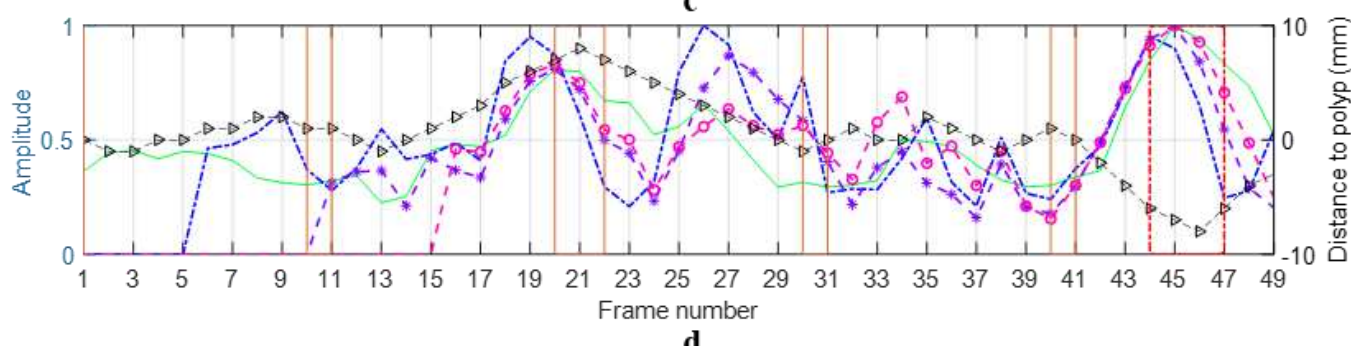

d

Figure 5. Comparison between AverageTotal strategy and the Hop\&NAverage with different $H$ and $N$ values. a) Trajectory 1 using Modified Monofocusing, b) Trajectory 1 using Bifocusing, c) Trajectory 2 using Modified Monofocusing, and d) Trajectory 2 using Bifocusing algorithm.

Regarding the focusing method, as shown in Figure 4, the difference in amplitude between the frames that correspond to healthy colon and those where the polyp is inside the detection zone of the device, is higher when Modified Monofocusing algorithm is used, allowing an easier detection of the polyp using a threshold without false positives. Figure 6 shows the cross-sectional reconstructed images using AverageTotal calibration and Modified Monofocusing and Bifocusing algorithms in the frame corresponding to the absolute maximum in Figure 4, that corresponds to the position of the polyp. The magnitude represented is the normalized reconstructed dielectric contrast profile at $7.6 \mathrm{GHz}$ represented in logarithmic scale between 0 and 1 . The blue circle in the middle of the plots indicates the position of the device and the red dots the position of each antenna. As shown in Figure 6 both algorithms are able to detect and locate the polyp showing higher intensities in the position of the polyp. Modified Monofocusing algorithm can obtain a punctual detection while Bifocusing does not obtain discrimination in range. 


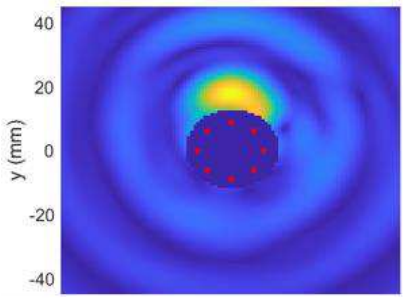

a

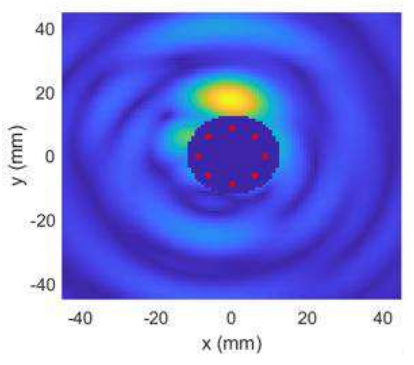

e

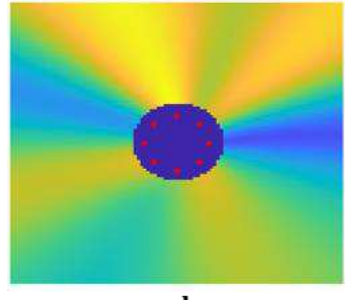

b

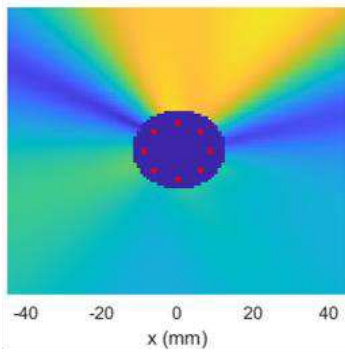

f

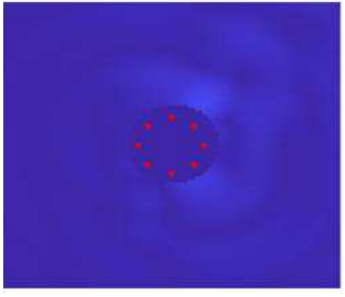

c

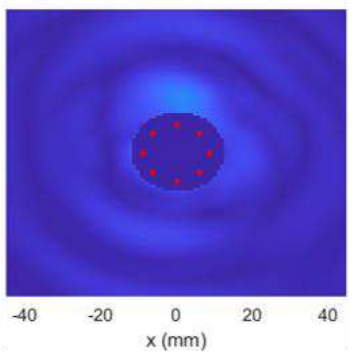

$\mathbf{g}$

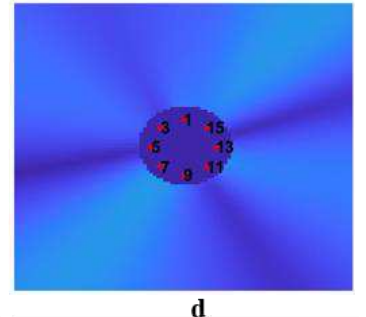

d

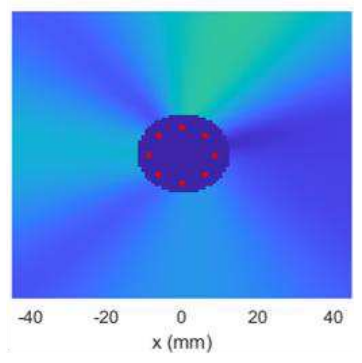

h

Figure 6 Cross-sectional reconstruction of the normalized dielectric contrast corresponding to Trajectories 1 and 2 for the frame that contains the absolute maximum amplitude of the trajectory and (polyp), and for a frame without polyps (healthy). a) Trajectory 1 using Modified Monofocusing with polyp, b) Trajectory 1 using Bifocusing with polyp, c) Trajectory 1 using Modified Monofocusing healthy, d) Trajectory 1 using Bifocusing healthy, e) Trajectory 2 using Modified Monofocusing with polyp, f) Trajectory 2 using Bifocusing with polyp, g) Trajectory 2 using Modified Monofocusing healthy, h) Trajectory 2 using Bifocusing healthy. The calibration has been performed with the AverageTotal strategy.

Finally, we assess the performance of the complete detection method for each calibration and focusing method (see Table 1). The detection thresholds have been defined to optimize the specificity and sensitivity to detect polyps from healthy mucosa. Table 1 shows the threshold as an interval, where the limits are the minimum and maximum values by which only the polyp is detected. The sensitivity and specificity have been calculated considering that a detection is valid if the threshold is satisfied at least in one of the frames where the polyp is inside the theoretical position of the polyp, that is [41, 44] for Trajectory 1 and $[44,47]$ for Trajectory 2. Results show that Modified Monofocusing algorithm provides a perfect detection without false positives, whereas Bifocusing algorithm presents a few false positives. With Modified Monofocusing the threshold intervals for Trajectory 1 and Trajectory 2 present an intersection of values, suggesting that it is possible to define a fixed threshold that would enable the detection of the polyp with both specificity and sensitivity equal to $100 \%$ irrespective of the trajectory. When using Bifocusing algorithm, no intersection between the thresholds of each calibration has been detected, meaning that the detection is not perfect. Table 1 also shows the fixed thresholds that are used to automatically detect the polyps in Trajectory 1 and Trajectory 2. In the case of Modified Monofocusing any value within the range $[3.45,3.89]$ would enable a perfect detection of the polyp, so the mean value of the interval, 3.67 , guarantees a sensitivity and specificity of $100 \%$ in the tested cases. In the case of Bifocusing, a threshold of 1.93 guarantees that polyps are detected but not specifically. It should be highlighted that the system is robust against the occurrence of false negatives since the sensitivity is always $100 \%$ and thus, it is not susceptible to miss polyps during colonoscopy.

\begin{tabular}{lllccccc}
\hline$\#$ & $\begin{array}{l}\text { Focusing } \\
\text { method }\end{array}$ & Calibration Strategy & $\begin{array}{c}\text { Threshold } \\
\text { interval }\end{array}$ & $\begin{array}{c}\text { Fixed } \\
\text { threshold }\end{array}$ & $\begin{array}{c}\text { Frames } \\
\text { detected }\end{array}$ & Specificity & Sensitivity \\
\hline 1 & MM & AverageTotal & {$[2.55,7.20]$} & 3.67 & {$[42,43]$} & $100 \%$ & $100 \%$ \\
1 & MM & Hop\&NAverage $(\mathrm{H}=3 \mathrm{~N}=15)$ & {$[2.75,7.37]$} & 3.67 & {$[42,43]$} & $100 \%$ & $100 \%$ \\
1 & MM & Hop\&NAverage $(\mathrm{H}=0 \mathrm{~N}=15)$ & {$[3.45,7.60]$} & 3.67 & 42 & $100 \%$ & $100 \%$ \\
\hline 1 & Bifocusing & AverageTotal & {$[1.83,2.07]$} & 1.93 & 42 & $100 \%$ & $100 \%$ \\
1 & Bifocusing & Hop\&NAverage $(\mathrm{H}=3 \mathrm{~N}=15)$ & {$[2.01,-]$} & 1.93 & 33,42 & $97.62 \%$ & $100 \%$ \\
1 & Bifocusing & Hop\&NAverage $(\mathrm{H}=0 \mathrm{~N}=15)$ & {$[2.02,2.07]$} & 1.93 & 33,42 & $97.62 \%$ & $100 \%$ \\
\hline 2 & MM & AverageTotal & {$[2.80,4.63]$} & 3.67 & {$[45,46]$} & $100 \%$ & $100 \%$ \\
2 & MM & Hop\&NAverage $(\mathrm{H}=3 \mathrm{~N}=15)$ & {$[3.26,4.14]$} & 3.67 & {$[44,46]$} & $100 \%$ & $100 \%$ \\
2 & MM & Hop\&NAverage $(\mathrm{H}=0 \mathrm{~N}=15)$ & {$[2.85,3.89]$} & 3.67 & {$[44,46]$} & $100 \%$ & $100 \%$ \\
\hline 2 & Bifocusing & AverageTotal & {$[1.99,2.22]$} & 1.93 & {$[20,21]$} & $95.56 \%$ & $100 \%$ \\
& & & & {$[44,47]$} & & $100 \%$ & $100 \%$
\end{tabular}




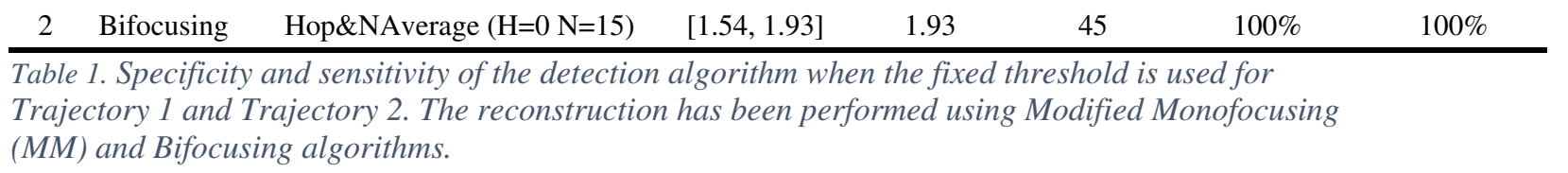

\section{Discussion}

We evaluated a novel microwave-based device and used the IDEAL model to drive this innovation. In this paper we report on the process of conceptualization and preparation before its implementation in patients. The IDEAL Framework ${ }^{20}$ and Recommendations represent a new paradigm for the evaluation of surgical operations, invasive medical devices and other complex therapeutic interventions. A miniaturized acquisition system for a microwave colonoscopy system to detect colorectal polyps with an alarm is presented in this paper. The acquisition device is designed as a switched cylindrical array of 16 antennas organized in two rings of eight antennas each containing the transmitters and the receivers respectively. The device is encapsulated and attached at the tip of bar simulating a colonoscope. The antennas are switched in pairs following a sequence and the transmission coefficients are measured to obtain a crosssectional image of the colon. This process is repeated a number of steps to form a trajectory. A vector network analyzer, a microcontroller and a laptop are used for data acquisition and signal processing.

The system has been designed to be compatible with colonoscopy and produce minimal changes into the current clinical practice. First of all, concerning size restrictions, the final dimensions of the acquisition device are $30 \mathrm{~mm}$ in length by $20 \mathrm{~mm}$ in diameter, having a total thickness of $3 \mathrm{~mm}$. The dimensions and shape of the device ensure non-obstruction of the front tip of the colonoscope, avoids injuring the patient or hindering the maneuverability of the colonoscope. The antenna elements show the typical electrically small antenna performance, characterized by a narrow bandwidth of $60 \mathrm{MHz}(0.8 \%$ fractional bandwidth) and an omnidirectional radiation pattern. Omnidirectional radiation pattern is suitable for this case where we want to ensure a $360^{\circ}$ coverage of the colon wall. Mutual coupling between adjacent antennas has been minimized to $-13 \mathrm{~dB}$ using cavity-backed antenna design.

The system is verified with a colon phantom that simulates a realistic colonoscopy exploration. The phantom models a section of a colon including haustrum and allows placement of different polyps. The phantom is composed of gelatin-oil based materials to mimic the dielectric properties of colon healthy mucosa and polyps with high grade dysplasia. Once manufactured, the tissue-mimicking materials have been measured with a coaxial probe achieving a dielectric properties very similar to real human tissues -relative permittivity of 41 and 46 , and conductivity of $9 \mathrm{~S} / \mathrm{m}$ and $9.8 \mathrm{~S} / \mathrm{m}$ respectively-. The properties remained stable during more than 40 days. The colon lumen has been created using casted expanded polyethylene and we drilled several holes to place spheres made of polyp-mimicking material. Even though the colon lumen model presented in this study is considered realistic as it contains haustrum, it does not include angulations. Therefore, further studies with a colon lumen model extracted from patient computed tomography colonography will be manufactured, measured and reconstructed in future experiments.

The acquired data is processed with a 3-step algorithm to emit an alarm when a polyp is detected. In the first step, the scattering fields are estimated by using three different calibration methods. This is a crucial step to remove the spatial-temporal uncertainty on the reception of the scattered fields due to the unknown and changing distance from the acquisition device and the colon wall. Next, two focusing methods are compared to obtain a cross-sectional image of each step, and finally, a detector based on a threshold is used to generate the alarm flag.

Regarding the calibration, the most important challenge is to differentiate the effect of the device movement from the effect of the presence of the polyp, as both produce changes in the measured scattered field. This can be observed if we compare the results of Trajectory 1 and Trajectory 2 in Figure 4 . For Trajectory 1 the difference between the absolute maximum, that corresponds to the polyp, and the local maximums, due to other effects, is lower than in case of Trajectory 2. Conversely, Trajectory 2 shows a smaller difference between absolute and local maximums suggesting that the main undesired effect that calibration must face is the device movement. This effect is evident in frames 15 to 22 of Trajectory 2, in this region, the accessory is moving along the $y$-axis towards the colon wall. Thus, the device movement is not completely corrected with any calibration, however, the correction obtained is sufficient to achieve a perfect detection of polyps in the studied cases. AverageTotal and Hop\&NAverage offer a similar performance to the Healthy Average Temporal Subtraction. Hence these two strategies can be used to 
simulate the Healthy Average Temporal Subtraction, which although it is the ideal calibration, it is unrealistic as it depends on prior knowledge of healthy frames, knowledge that is not available in a clinical scenario. AverageTotal offers similar results to Healthy Average Temporal Subtraction if the percentage of frames with polyp vs healthy frames is less than $10 \%$. Hop\&NAverage seems to be the most suitable calibration in a real case, even though the first $N+H$ frames cannot be calibrated nor analyzed. Moreover, the selection of $N$ values is a key, as the bigger $N$ is, the better the results, but the more the calibrated detection is delayed. With these trajectories the best results are given for $N=15$ and $H=3$. The optimization of these values should be done by analyzing more trajectories in future experiments.

The difference between both imaging algorithms, Modified Monofocusing and Bifocusing, is clear. Modified Monofocusing presents better results when avoiding false positives in regions with big movements. Moreover, Modified Monofocusing presents a focused detection with less artifacts, whereas Bifocusing is only able to focus on an angular section. The main reason for this lack of range resolution is the narrow frequency band of the antennas. However, this is not a problem for our system, since only an alarm is provided to the doctor that will be only active during the frames the polyp is detected by the algorithm. According to the opinion of more than 30 doctors with whom we have collaborated, it is not necessary for our device to provide angular information on the position of the polyp as it could distract the doctor's attention during colonoscopy. The unusual imaging configuration in which the antennas are surrounded by the imaging region (colon) means that half of the transmitting and receiving antennas in the array cannot see all the points of the imaging region, in particular, if we define two hemispheres in the reconstruction area, where the equator is the line that joins two antennas $180^{\circ}$ apart, when the point we want to focus is not on the same hemisphere as the antenna, there will be no vision possible since the signal cannot pass through the device. For this reason, focusing based on re-establishing the effect produced in the fields due to the distance between an image point and the transmitting and receiving antennas is wrong for these antenna combinations. This effect has been reduced by only including in the reconstruction the three closest receiving antennas for each transmitter. The residual error produced by this effect is translated in artifacts in the reconstructed image especially appearing with Bifocusing method. Moreover, due to the narrowband behavior of the antennas, we cannot benefit from frequency averaging that is commonly used to cancel frequency selective $\operatorname{artifacts}^{21}$.

Finally, it has been observed that it is possible to define a threshold that would enable an automatic detection of the polyp in the trajectories studied, regardless of the calibration strategy employed. Notwithstanding, more trajectories must be tested and more sophisticated thresholding methods will be designed to make the threshold more robust.

\section{Methods}

Microwave imaging is based on illuminating the object under test, the phantom, with an incident radiation, $E^{i}$, and measuring the total received fields, $E^{t}$, resulting from the interaction of the incident radiation and the body under test. The total received field is the superposition of the incident field and the scattered field, $E^{s}$, that contains the information of the spatial changes of the dielectric properties of the phantom. By processing the total field with an imaging algorithm, the dielectric property contrast of the phantom can be reconstructed.

\section{Data acquisition system}

Scattered field is proportional to the transmission coefficient. In the proposed system the transmission coefficients are measured using a combination of hardware and software components. The hardware is composed by a switched antenna array integrated in the acquisition device, a microprocessor (Arduino Nano), a 2-port vector network analyzer (VNA) and a laptop. The VNA ports and the microcontroller are connected to the acquisition device through coaxial cables, which in turn is connected to a laptop to take synchronized measurements. The microcontroller is used to configure the two single-pole-eight-throw (SP8T) radiofrequency switches that select the active pair of transmitting and receiving antennas. Prior to the measurement, the VNA is calibrated up to the point of antenna feeding connection. The standard VNA calibration system including open-short-load connections is adopted. Since the acquisition device will be provided as a disposable set that includes the antenna array, the switching network and the cables; the VNA calibration only gets rid of the effects of the VNA. Hence, the effect of slim and long connecting coaxial cables remains and reduces the efficiency of the system. The software is a Matlab code running in a laptop that performs 5 steps: (1) Establish the connection between the VNA and the 
microcontroller and configure the measurement (bandwidth of $350 \mathrm{MHz}, N_{f}=801$ frequency points, transmitting power of $-5 \mathrm{dBm}$ ), (2) select one transmitting and one receiving antenna pair, (3) send the transmitting and receiving antenna selection code to the microcontroller, (4) send trigger signal to the VNA to start the measurement of the transmitting coefficient between active antennas along the selected frequency band, (5) read the measured data from the VNA, (6) repeat (2)-(5) until the data from all the antenna combinations are measured, (7) move the acquisition device to the next position of the trajectory using the positioning system to obtain data from the next colon cross-section. The collected data is stored for processing. In total 24 combinations of transmitting and receiving antennas are measured for each step of the trajectory. These combinations are obtained by alternatively selecting for each transmitting antenna the three closest receiving antennas that are the adjacent one, and the two diagonal ones.

To simulate a real colonoscopy with the phantom, we have taken as a reference a real colonoscopy trajectory ${ }^{22}$. A trajectory refers to the $(\mathrm{x}, \mathrm{y}, \mathrm{z})$ coordinates of the centroid of the acquisition cylindrical device, where XY-plane is a horizontal or cross-sectional plane of the colon lumen, and $\mathrm{z}$ is the vertical coordinate. The origin of coordinates $(0,0,0)$ is defined when the device is situated at the center of the cross-sectional plane of the colon lumen and the center of the 2 antenna rings is at the same height as the top base of the colon lumen (see Figure 7). In this study, two different trajectories are presented: one rectilinear $(\mathrm{x}=0, \mathrm{y}=-8 \mathrm{~mm})$ of $184 \mathrm{~mm}$ in length that represents an ideal case in which the position of the device in $\mathrm{x}$ and $\mathrm{y}$ axis is maintained along the trajectory, and a realistic trajectory of $172 \mathrm{~mm}$ in length in which the device is displaced in the y-axis. Each step of the trajectory is called a frame, $E_{z_{i}}^{t}(\vec{r}, f)$ being $i$ the index of the current frame. Each frame of the trajectories is separated $4 \mathrm{~mm}$ from the subsequent one along the z-axis. For each frame a $N_{a} \times 3 \times N_{f}$ matrix is obtained, being $\vec{r}=(x, y)$ and $N_{a}=8$ is the number of transmitting and receiving antennas.

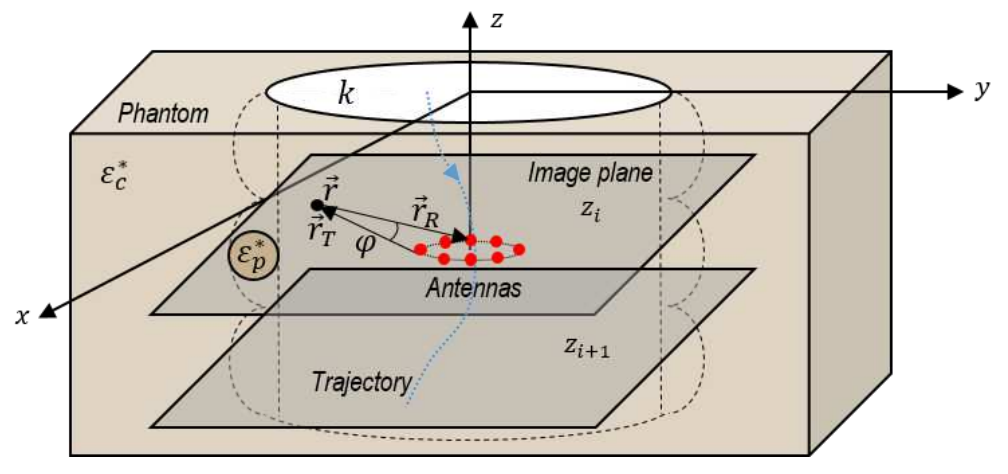

Figure 7. Trajectory described by the antenna array along the colon phantom.

\section{Signal and image processing algorithm}

Firstly, the data acquisition software is initialized and the VNA configuration parameters are imported inside the software. Next the transmission coefficients' matrices from all the measurement steps of the trajectory are loaded. For each step of the trajectory the following steps are conducted: (1) calibrate the data to remove any error due to the movement of the device and the colon wall effect, (2) focus the data to obtain the reconstructed image of the dielectric contrast profile, (3) evaluate the threshold to identify whether there is a polyp or not, (4) repeat (1)-(3) for each step of the trajectory, and (5) calculate the overall sensitivity and specificity of the detection algorithm for the trajectory.

\section{Calibration}

Calibration consists of isolating solely the scattered field from the polyp, removing therefore, any other effect. In our case the main effect that contaminates the desired signal is the movement of the device and thus the effect of having the colon wall at unknown and varying distances. We propose three calibration methods that share the same principle that is to subtract from the actual measured frame, a previous or a combination of previous measurement frames. The ideal calibration consists of subtracting from the scattered field measured in a phantom with a polyp, its healthy counterpart, that is a measurement with the same phantom but without polyps. In a real situation, this kind of calibration is unfeasible because the healthy version of the actual colon under test is not available. Therefore, in this paper three different methods to estimate the scattered field due to healthy colon are studied.

The first calibration approach is called Healthy Average Temporal subtraction (HAT). This calibration consists on calculating the average of the transmission coefficient's matrices corresponding to healthy 
frames, $E_{z_{h, j}}^{t}(\vec{r}, f)$, and subtracting it to the current measurement frame, $E_{z_{i}}^{t}(\vec{r}, f)$. This is possible in phantom experiments because there is prior knowledge of where the polyp is, but it is not feasible in al clinical setting.

$$
E_{z_{i}, H A T}^{s}(\vec{r}, f)=E_{z_{i}}^{t}(\vec{r}, f)-\sum_{j=1}^{N_{h}} \frac{1}{N_{h}} E_{z_{h, j}}^{t}(\vec{r}, f)
$$

Where $N_{h}$ is the number of healthy steps averaged. The second calibration approach is called Average Total (AT) and consists of averaging all the trajectory measurement steps and subtracting them to each measurement frame. This method is based on the hypothesis that in a colonoscopy the vast majority of frames are healthy.

$$
E_{z_{i}, A T}^{s}(\vec{r}, f)=E_{z_{i}}^{t}(\vec{r}, f)-\sum_{i=1}^{N_{z}} \frac{1}{N_{z}} E_{z_{i}}^{t}(\vec{r}, f)
$$

Where $N_{z}$ is the total number of steps. The last method is called Hop and N Average Temporal (HNAT), the idea behind this method is to skip the $N$ closest frames to the current frame. This is important in case a polyps is present since the effect of its presence is not included in the calibration if $N$ is sufficiently large. In this case, the scattered field produced by the polyp is estimated as:

$$
E_{z_{i}, H N A T}^{s}(\vec{r}, f)=E_{z_{i}}^{t}(\vec{r}, f)-\sum_{j=i-H}^{i-H-N} \frac{1}{N} E_{z_{j}}^{t}(\vec{r}, f)
$$

Being $H$ the number of steps that includes the leap and $N$ the number of steps that are averaged.

\section{Focusing}

Due to the small distances between the antennas and the colon wall, the majority of the power transmitted by the antenna propagates through and reflected by a slice of the colon with negligible reflections from other slices. Therefore, a two-dimensional electromagnetic analysis can be performed to obtain a reasonable estimation of the image profile. Considering the constraints of the system, two versions of a frequency domain imaging algorithm are implemented, namely Bifocusing (B) and Modified Monofocusing (M), respectively, see equations (4) and (5.) These algorithms form every image point of the dielectric property contrast of the colon by creating one or two focused groups of antennas (one for the transmitters and the other for the receivers). The received scattered field from each antenna pair is numerically weighted with a focusing operator so as to be focused on a unique point in the reconstruction grid. The focusing operator restores the module and phase changes suffered by a wave on its way to and/or from every image point. For each image point, the coherent summation of all the scattering focused fields from all antenna combinations results in a large intensity value if the actual point is the origin of the scattering (polyp). If not, the summation results in a small value and this contribution can be considered as noise. Note that we assumed that the $\mathrm{z}$-coordinate of the transmitting and receiving rings of antennas is the same and thus its position is denoted as $\vec{r}_{T}=\left(x_{T}, y_{T}\right)$ and $\vec{r}_{R}=\left(x_{R}, y_{R}\right)$ respectively.

$$
\begin{gathered}
I_{z_{i}}^{B}(\vec{r})=\left|\sum_{k=(j-1)_{N_{a}}} \sum_{j=1}^{N_{a}} \frac{E_{s}\left(\vec{r}_{T_{j}}, \vec{r}_{R_{k}}, z_{i}\right)}{k^{2} H_{0}^{2}\left(k\left|\vec{r}_{T_{j}}-\vec{r}\right|\right) H_{0}^{2}\left(k\left|\vec{r}_{R_{k}}-\vec{r}\right|\right)}\right| \\
I_{z_{i}}^{M M}(\vec{r})=\left|\sum_{k=(j-1)_{N a}} \sum_{j=1}^{N_{a}} E_{s}^{2}\left(\vec{r}_{T_{j}}, \vec{r}_{R_{k}}, z_{i}\right) J_{1}^{2}\left(k\left|\vec{r}_{R_{k}}-\vec{r}\right|\right) e^{j 2\left(k\left|\vec{r}_{R_{k}}-\vec{r}\right|+\varphi\right)}\right|
\end{gathered}
$$

Being $k=2 \pi f$ the wavenumber and $\varphi$ the angle between the transmitting and the receiving antenna.

\section{Thresholding}

For each reconstructed image frame, $I_{z_{i}}(\vec{r})$, the thresholding method compares the maximum of the current reconstruction with the average maximum of the previous reconstructed images that have been classified as healthy, $I_{z_{h, j}}(\vec{r})$. If the quotient of those values is higher than the detection threshold, the current frame is labeled as containing a polyp (Equation (6)). Otherwise, the current frame is labeled as 
healthy and is added to the average of healthy maximums. The first frame is always considered as healthy. For the frames labeled as containing a polyp, the system will emit an alarm to warn the endoscopist.

$$
\max \left\{I_{z_{i}}(\vec{r})\right\} / \sum_{j=1}^{N_{h}} \frac{1}{N_{h}} \max \left\{I_{z_{h, j}}(\vec{r})\right\}>\text { threshold }
$$

Finally, the overall performance of the system to detect polyps in all the trajectory is evaluated using sensitivity and specificity. The sensitivity, also called the true positive (TP) rate, measures the percentage of cases having a polyp that are correctly diagnosed as having the lesion. A false negative (FN) occurs when a negative result is reported to a trajectory that does have a polyp. The specificity, also called the true negative $(\mathrm{TN})$ rate, measures the percentage of healthy cases that are correctly identified as not having any polyp. A false positive (FP) is reported when the test wrongly indicates that a polyp is present. The values of sensitivity and specificity are related to TP, FP, TN, and FN values through the following equations:

$$
\begin{aligned}
& \text { sensitivity }=\frac{T P}{T P+F N} \\
& \text { specificity }=\frac{T N}{T N+F P}
\end{aligned}
$$

\section{Phantom creation and measurement}

To manufacture the tissue-mimicking materials we adapted a recipe of oil-gelatin based phantoms developed for breast tissues ${ }^{23}$ to the dielectric properties of healthy colon mucosa and polyps with high grade dysplasia ${ }^{16}$. Oil-gelatin materials are low-cost, easy to produce, non-toxic and it is easy to change their dielectric properties by modifying the proportion of oil and water. In this case, we increased the amount of oil to produce the high dielectric properties of healthy colon mucosa and polyps. Besides, some steps have been added to the original protocol ${ }^{23}$ to be able to produce high quality large batches of material. First of all, the gelatin was previously hydrated with cold deionized water to completely dissolve the gelatin and improve the homogeneity of the final mixture. To increase the velocity of polymerization and avoid deposition of solids, the mixture is stirred continuously in a cold water bath until their temperature falls to $29^{\circ} \mathrm{C}$, then the material is poured in the final container and the phantom is stored in the fridge until it is completely solid. Finally, the phantom was preserved in the fridge hermetically closed to avoid dehydration and to keep a solid consistency. The composition of phantom mixtures is summarized in Table 2.

\begin{tabular}{lllll}
\hline Tissue & $\begin{array}{l}\text { Deionized water } \\
(\mathbf{m l})\end{array}$ & $\begin{array}{l}\text { Gelatin 250 } \\
\text { bloom }(\mathbf{g})\end{array}$ & $\begin{array}{l}\text { Sunflower oil } \\
(\mathbf{m l})\end{array}$ & $\begin{array}{l}\text { Dishwashing } \\
\text { liquid }(\mathbf{m l})\end{array}$ \\
\hline Healthy mucosa & 3260.76 & 326.62 & 1221.43 & 182.76 \\
Adenoma HGD & 108.12 & 10.83 & 20.4 & 6.06 \\
\hline
\end{tabular}

Table 2. Composition of phantom mixtures.

\section{Data availability}

The datasets generated and analyzed during the current study are available from the corresponding author on reasonable request.

\section{References}

1. Bray, F. et al. Global cancer statistics 2018: GLOBOCAN estimates of incidence and mortality worldwide for 36 cancers in 185 countries. CA. Cancer J. Clin. 68, 394-424 (2018).

2. Siegel, R. L., Miller, K. D. \& Jemal, A. Cancer statistics, 2015. CA. Cancer J. Clin. 65, 5-29 (2015).

3. Surveillance Research Program, National Cancer Institute SEER*Stat software. (2021). Available at: seer.cancer.gov/seerstat.

4. van Rijn, J. C. et al. Polyp miss rate determined by tandem colonoscopy: a systematic review. Am J Gastroenterol 101, 343-350 (2006). 
5. Samadder, N. J. et al. Characteristics of missed or interval colorectal cancer and patient survival: a population-based study. Gastroenterology 146, 950-960 (2014).

6. Ngu Sing, W. \& Rees, C. Can technology increase adenoma detection rate? Therap. Adv. Gastroenterol. 11, 1-18 (2018).

7. Urban, G. et al. Deep Learning Localizes and Identifies Polyps in Real Time With 96\% Accuracy in Screening Colonoscopy. Gastroenterology 155, 1069-1078.e8 (2018).

8. Freedman, D. et al. Detecting Deficient Coverage in Colonoscopies. IEEE Trans. Med. Imaging XX, 1-1 (2020).

9. Nikolova, N. K. Introduction to Microwave Imaging. (Cambridge University Press, 2017). doi: $10.1017 / 9781316084267$

10. Meaney, P. M. et al. Clinical microwave tomographic imaging of the calcaneus: a first-in-human case study of two subjects. IEEE Trans. Biomed. Eng. 59, 3304-13 (2012).

11. Semenov, S. Y. et al. Microwave Tomographic Imaging of the Heart in Intact Swine. J. Electromagn. Waves Appl. J. Electromagn. Waves Appl 207, 873-890 (2006).

12. Shere, M. et al. MARIA® M5: A multicentre clinical study to evaluate the ability of the Micrima radio-wave radar breast imaging system (MARIA®) to detect lesions in the symptomatic breast. Eur. J. Radiol. 116, 61-67 (2019).

13. O’Loughlin, D. et al. Microwave breast imaging: Clinical advances and remaining challenges. IEEE Trans. Biomed. Eng. 65, (2018).

14. Crocco, L., Karanasiou, I., James, M. L. \& Conceição, R. C. Emerging electromagnetic technologies for brain diseases diagnostics, monitoring and therapy. Emerging Electromagnetic Technologies for Brain Diseases Diagnostics, Monitoring and Therapy (Springer International Publishing, 2018). doi:10.1007/978-3-319-75007-1

15. Karadima, O. et al. Experimental Validation of Microwave Tomography with the DBIM-TwIST Algorithm for Brain Stroke Detection and Classification. (2020). doi:10.3390/s20030840

16. Guardiola, M. et al. Dielectric properties of colon polyps, cancer, and normal mucosa: Ex vivo measurements from 0.5 to $20 \mathrm{GHz}$. Med. Phys. 45, 3768-3782 (2018).

17. Klemm, Maciej, et al. Radar-Based Breast Cancer Detection Using a Hemispherical Antenna Array-Experimental Results - IEEE Journals \&amp; Magazine. EEE Trans. antennas Propag. 57, 1692-1704 (2009).

18. Mohammed, B. J., Abbosh, A. M., Member, S., Mustafa, S. \& Ireland, D. Microwave System for Head Imaging. 63, 117-123 (2014).

19. Guardiola, M. et al. Design and evaluation of an antenna applicator for a microwave colonoscopy system. IEEE Trans. Antennas Propag. 67, (2019).

20. IDEAL Collaboration. Available at: https://www.ideal-collaboration.net/.

21. Jofre, L. et al. UWB Tomographic Radar Imaging of Penetrable and Impenetrable Objects. doi:10.1109/JPROC.2008.2008854

22. Jagadeesan Jayender, Raul San Jose Estepar, K. G. V. New Kinematic Metric for Quantifying Surgical. in Information Processing in Computer-Assisted Interventions. IPCAI 2010. Lecture Notes in Computer Science 81-90 (2010).

23. Di Meo S, Pasotti L, Iliopoulos I, Pasian M, Ettorre M, Zhadobov M, M. G. Tissue-mimicking materials for breast phantoms up to $50 \mathrm{GHz}$. Phys Med Biol. 5, (2019).

\section{Acknowledgements}

A.G. acknowledges the financial support from DIN2019-010857, J.R. acknowledges the financial support from PID2019-107885GB-C31/AEI/10.13039/501100011033, and R.S., M.G., and I.B. acknowledges the financial support from the European Union's Horizon 2020 research and innovation programme under grant agreement No 960251. 


\section{Author contributions}

R.S. and M.G. contributed in the development of the colon imaging system, designed, simulated and prototyped the acquisition device. S.M. and M.G. fabricated the realistic colon phantom. A.G. and W.D. developed the imaging software. G.F. contributed to the concept of the realistic colon phantom, validated the design and results in preclinical scenario. J.R. contributed to the antenna applicator concept. A.G., R.S., W.D., S.M. and M.G. wrote the main manuscript text and prepared all the figures. M.G. supervised the whole project. All authors reviewed the manuscript.

\section{Competing interests}

A.G., R.S., W.D., S.M, G.F.E., I.B. and M.G. are employees of MiWEndo Solutions S.L., J.R., G.F.E, I.B. and M.G. are also shareholders of the company. 


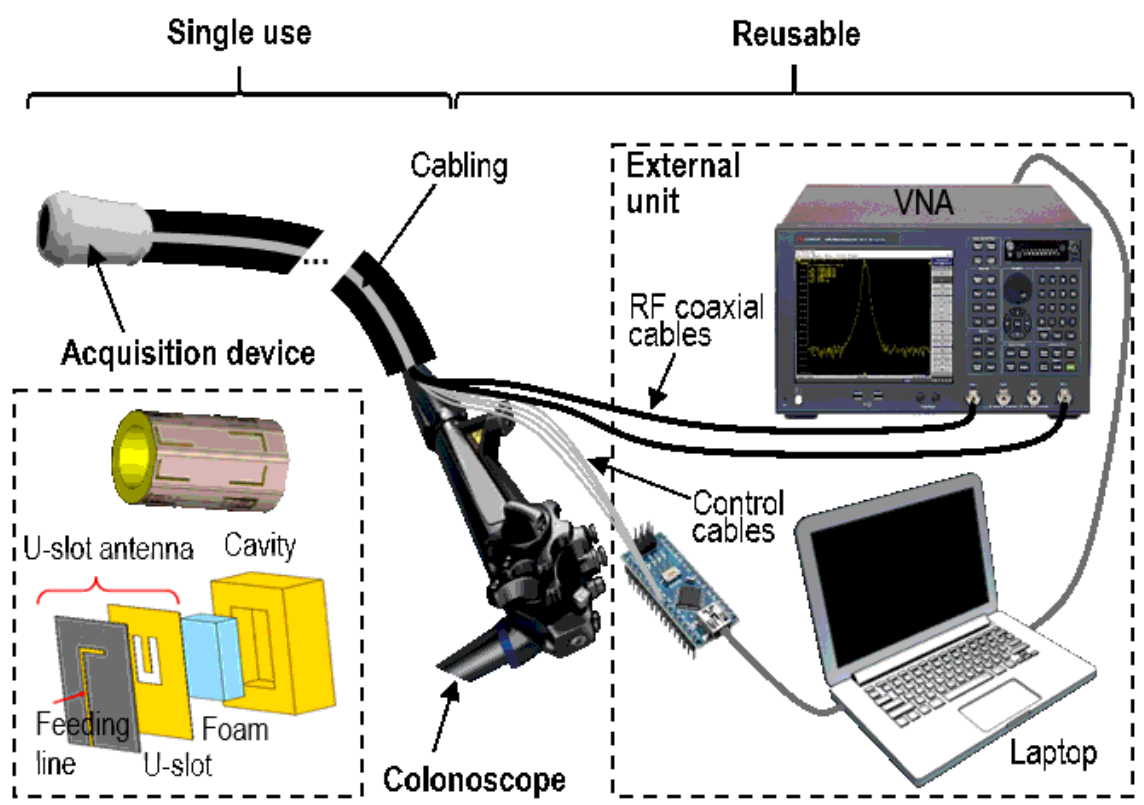

a

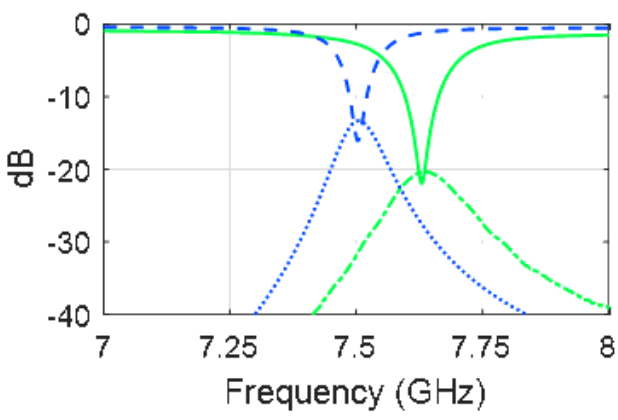

-Measured reflection parameter - - -Simulated reflection parameter -----Measured transmission parameter Simulated transmission parameter

\section{Figure 1}

(a) Imaging system consists of a cylindrical ring-shaped acquisition device attached to the tip of a colonoscope connected via cables to the external unit. The external unit consists of a vector network analyzer, a microcontroller and a laptop. (b) Comparison between the measured and simulated reflection and transmission coefficients.

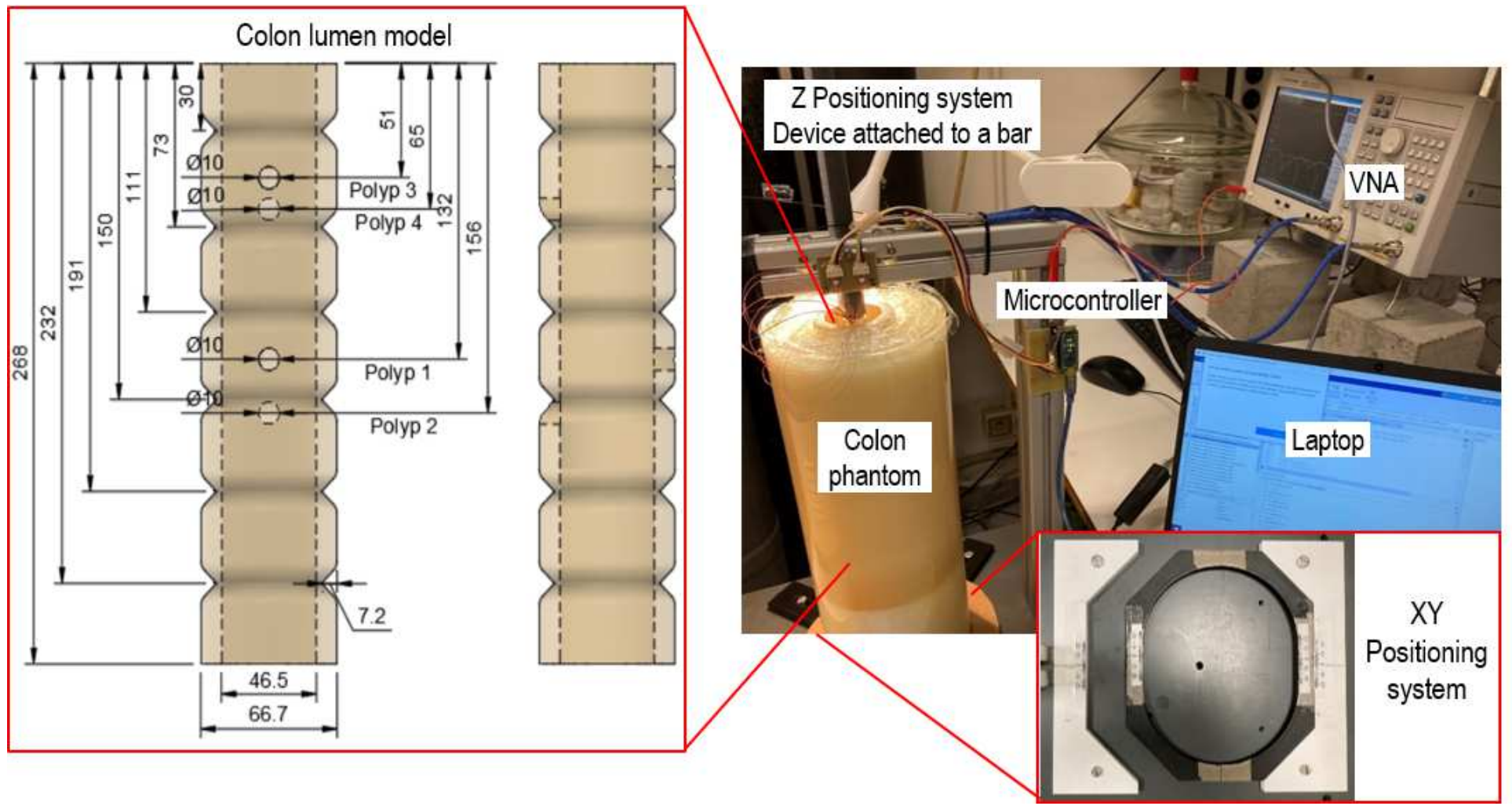




\section{Figure 2}

Measurement setup. The acquisition device is attached to the tip of a plastic bar. The colon phantom is placed on a 3D positioning system. The colon phantom is composed by tissue-mimicking materials poured in a methacrylate cylindrical box and an expanded polystyrene colon lumen model. The external microwave transceiver, controlling and processing unit is composed by a vector network analyser (VNA), a microcontroller, a laptop.

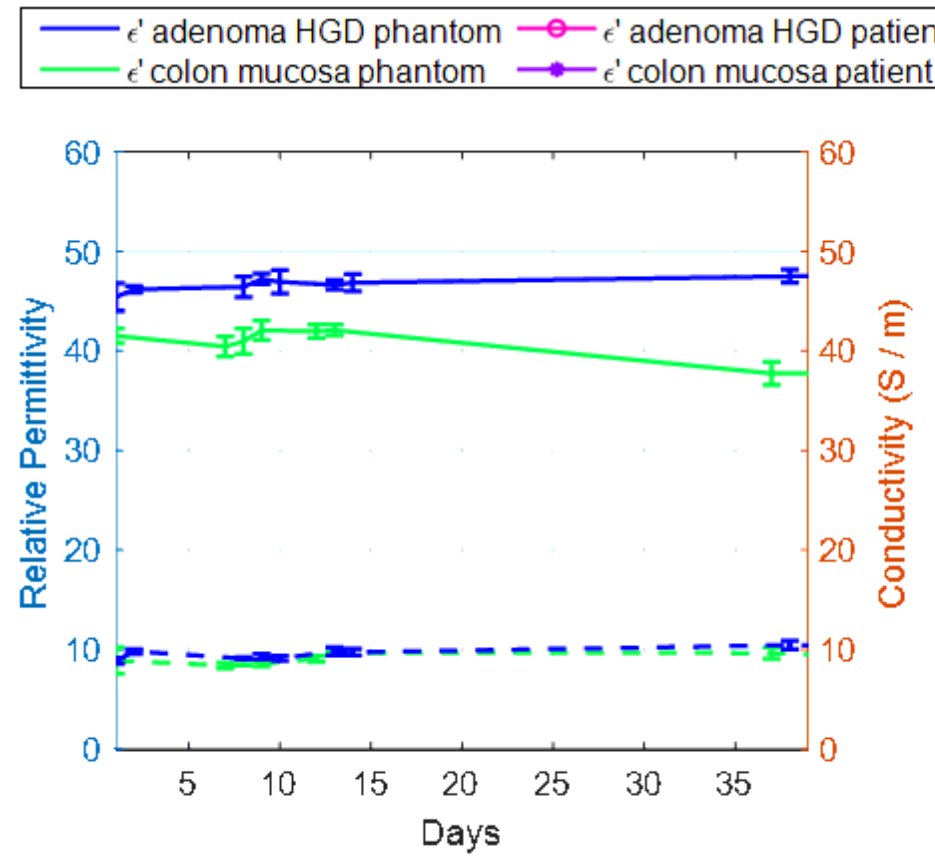

a

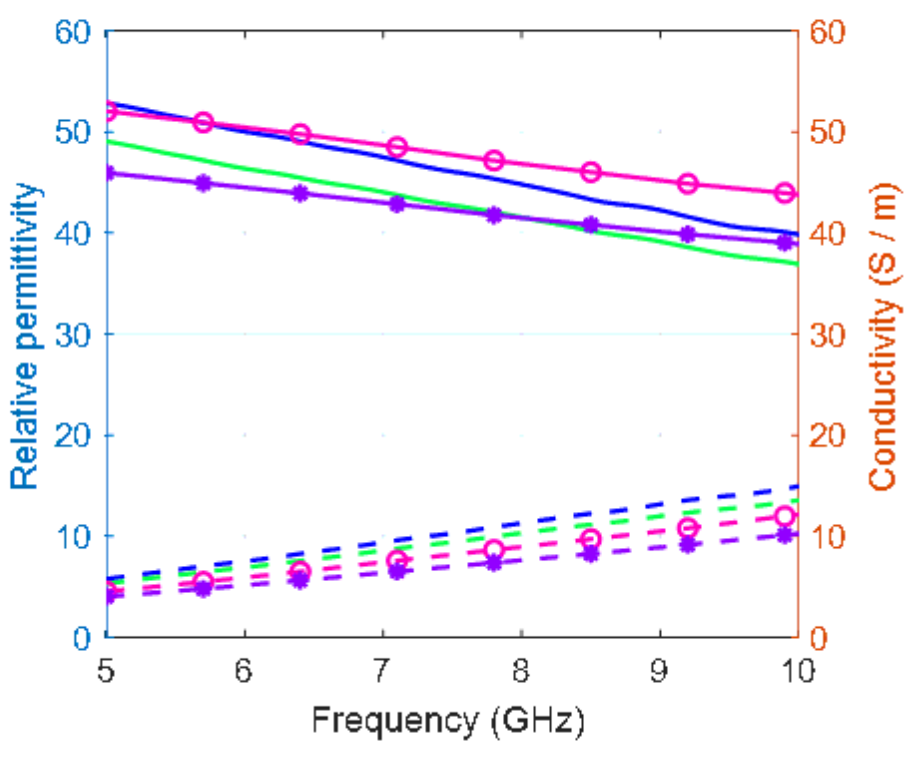

b

\section{Figure 3}

Dielectric properties of the tissue-mimicking materials used for the phantom. (a) time evolution of the average and standard deviation of the measured relative permittivity $\left(\nabla^{\wedge^{\prime}}\right)$ and conductivity $(\sigma)$ of the adenoma with high grade dysplasia's (HGD) phantom and the colon mucosa's phantom materials at 7.6 $\mathrm{GHz}$, (b) measured relative permittivity and conductivity of the phantom compared to the real patient tissues over the frequency. 

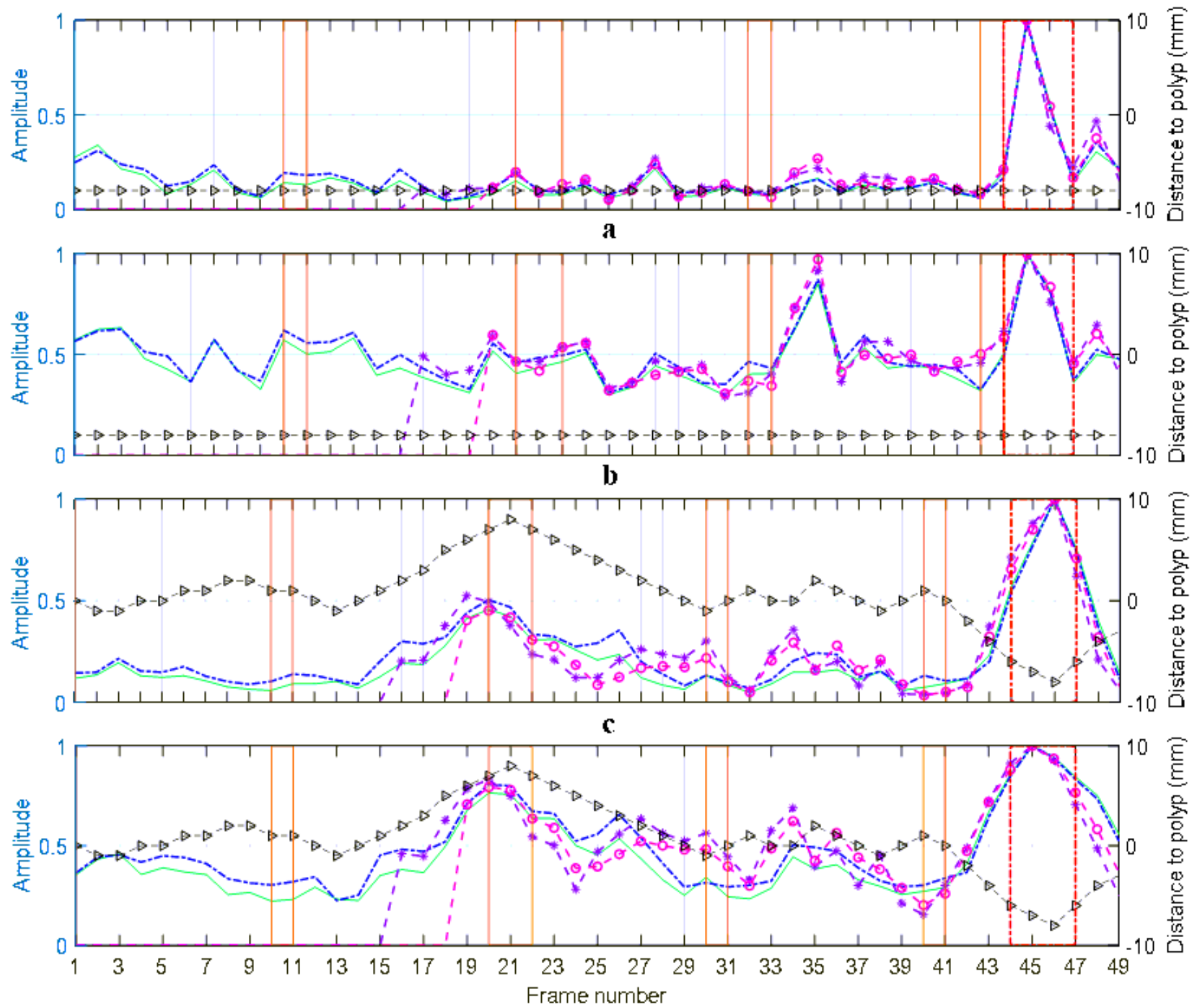

d

\section{Figure 4}

Evolution of the normalized maximum amplitude registered in each frame of the trajectories after applying the different calibration and focusing strategies proposed. a) Trajectory 1 using Modified Monofocusing, b) Trajectory 1 using Bifocusing, c) Trajectory 2 using Modified Monofocusing, and d) Trajectory 2 using Bifocusing algorithm. 

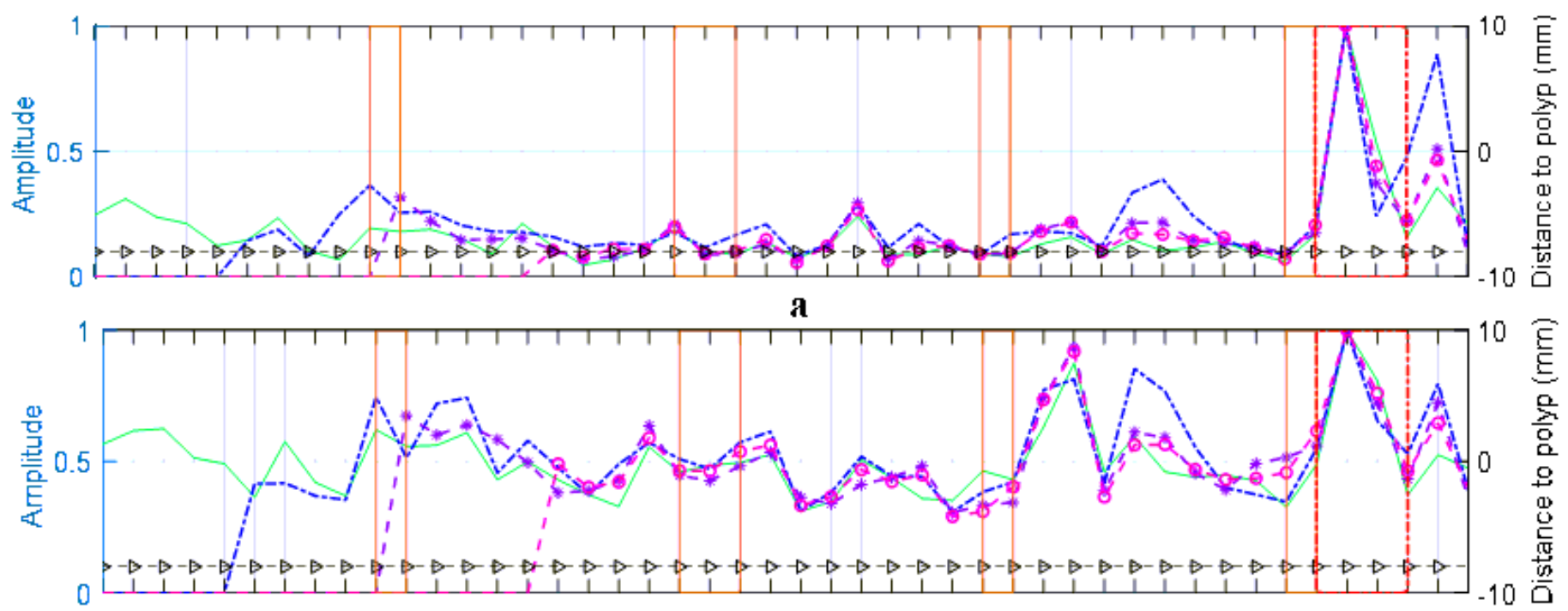

b
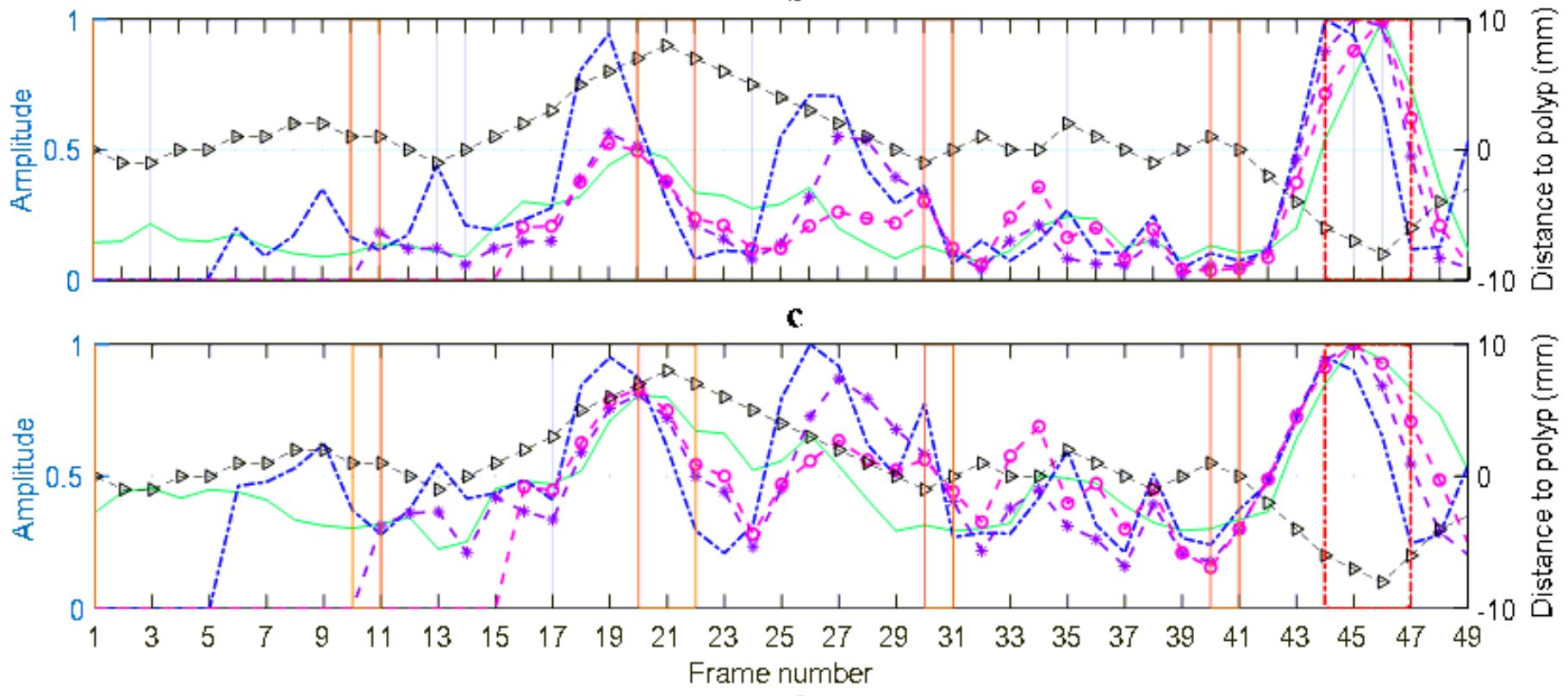

d

Figure 5

Comparison between AverageTotal strategy and the Hop\&NAverage with different $\mathrm{H}$ and $\mathrm{N}$ values. a) Trajectory 1 using Modified Monofocusing, b) Trajectory 1 using Bifocusing, c) Trajectory 2 using Modified Monofocusing, and d) Trajectory 2 using Bifocusing algorithm. 

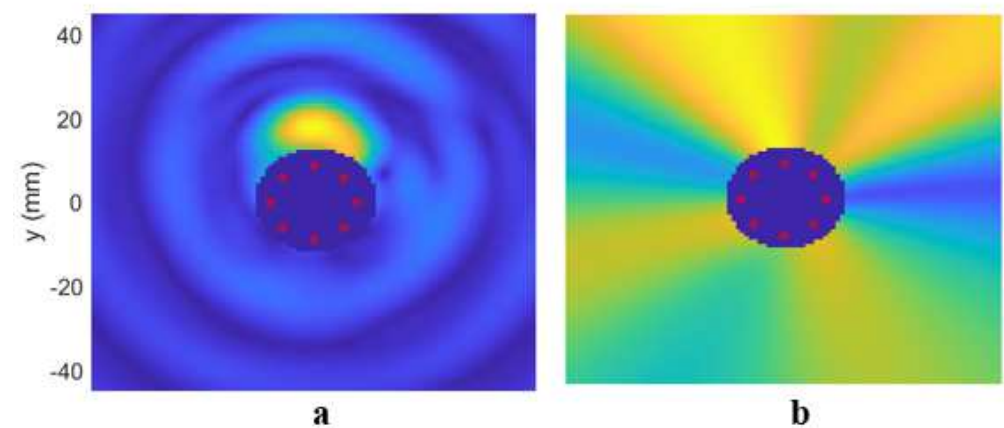

b
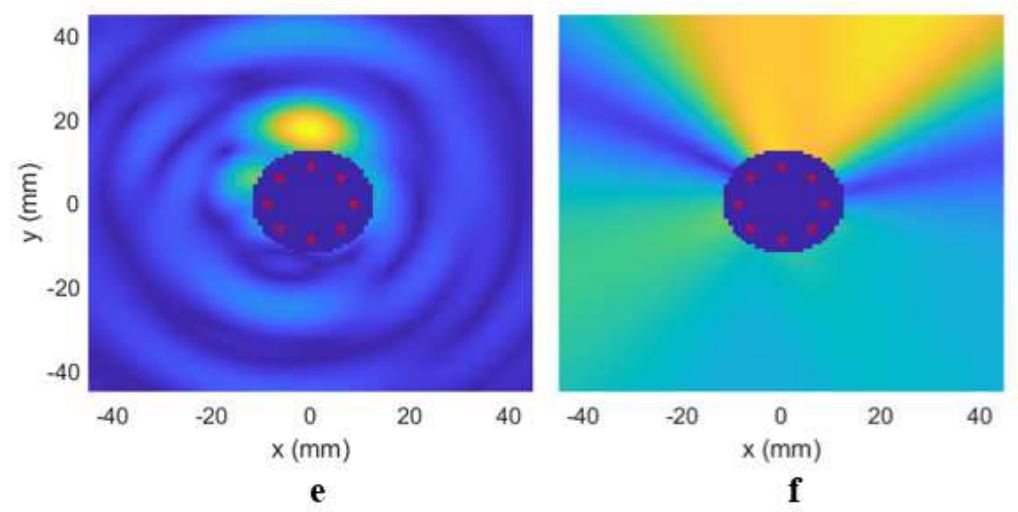

f

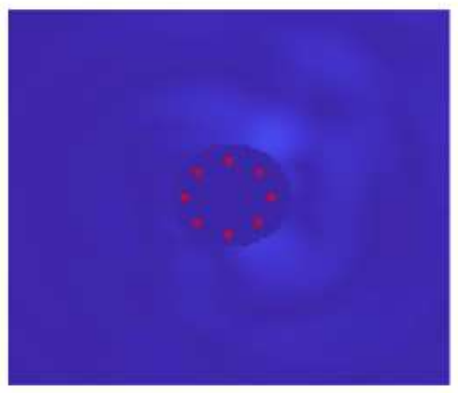

c

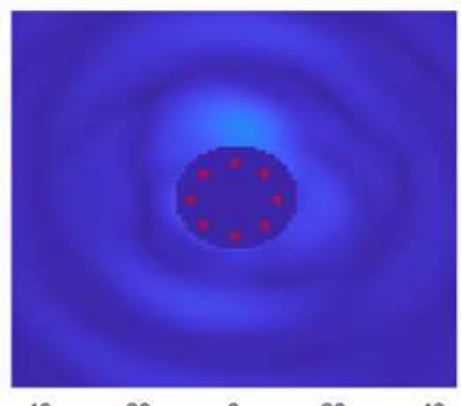

$-40$
$-20$ $x(\mathrm{~mm})$

g

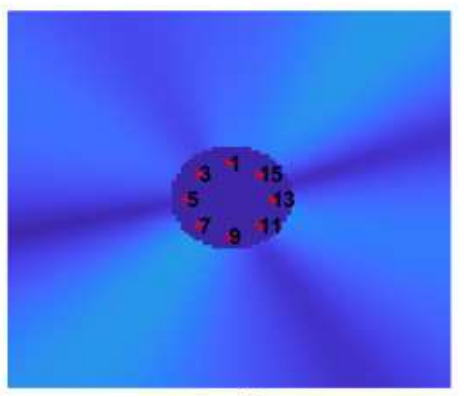

d

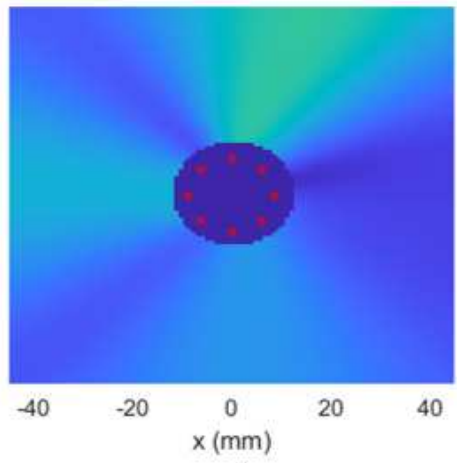

h

\section{Figure 6}

Cross-sectional reconstruction of the normalized dielectric contrast corresponding to Trajectories 1 and 2 for the frame that contains the absolute maximum amplitude of the trajectory and (polyp), and for a frame without polyps (healthy). a) Trajectory 1 using Modified Monofocusing with polyp, b) Trajectory 1 using Bifocusing with polyp, c) Trajectory 1 using Modified Monofocusing healthy, d) Trajectory 1 using Bifocusing healthy, e) Trajectory 2 using Modified Monofocusing with polyp, f) Trajectory 2 using Bifocusing with polyp, g) Trajectory 2 using Modified Monofocusing healthy, h) Trajectory 2 using Bifocusing healthy. The calibration has been performed with the AverageTotal strategy. 


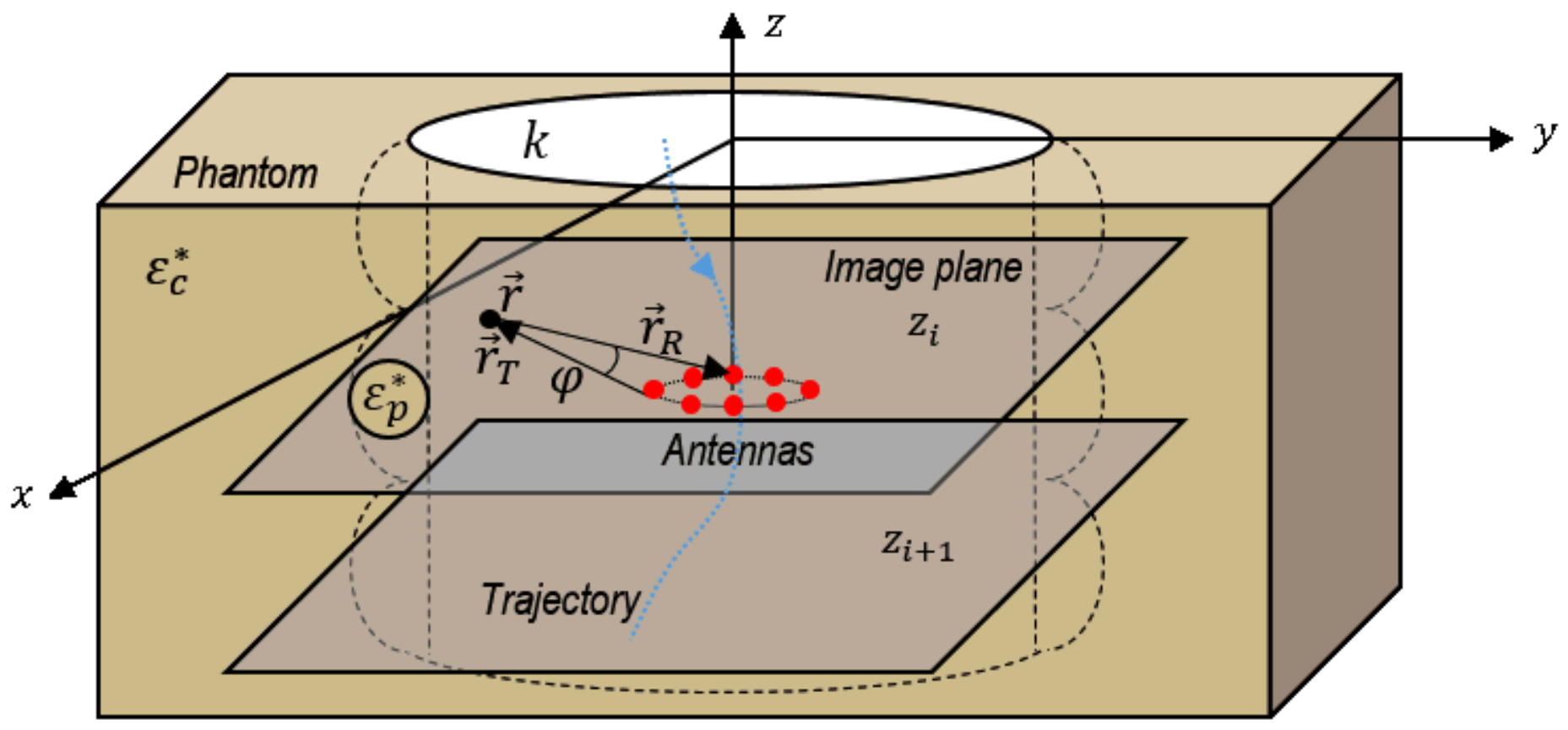

Figure 7

Trajectory described by the antenna array along the colon phantom. 